\title{
Transcriptome analysis and cytokine profiling of naive $T$ cells stimulated by a tumor vaccine via CD3 and CD25
}

\author{
PHILIPPE FOURNIER, MAXIMILIAN AIGNER and VOLKER SCHIRRMACHER ${ }^{1}$ \\ German Cancer Research Center, Heidelberg, Germany \\ Received May 28, 2010; Accepted July 19, 2010 \\ DOI: 10.3892/ijo_00000796
}

\begin{abstract}
T-cell receptor engagement by peptide/MHC complexes constitutes the main signal for the activation of naive $\mathrm{T}$ cells, but for a productive generation and maintenance of effector cells, full activation requires additional signals driven by costimulatory molecules present on activated antigenpresenting cells. Herein we describe T cell costimulation via CD25, the interleukin (IL)-2 receptor, during priming of naive $\mathrm{T}$ cells with a tumor vaccine. To this end, we produced, purified and characterized the fusion protein bsHN-IL2 which contains the IL-2 cytokine and an antibody scFv fragment directed towards the Hemagglutinin-Neuraminidase (HN) protein of Newcastle Disease Virus (NDV). Tumor vaccine cells were modified by infection with this virus which allows the attachment of the immunocytokine bsHN-IL2. In the presence of CD3-mediated signal 1, the vaccine/bsHN-IL2 provided via CD25 a strong bystander antitumor effect in vitro leading to tumor growth inhibition, even stronger than the vaccine/bsHN-CD28 which provides costimulation via CD28. Transcriptome analysis of naive T cells which were stimulated with the vaccine/bsHN-IL2 showed, similarly to the vaccine/ bsHN-CD28, upregulation of 71 genes belonging to different signalling pathways, including PLC- $\gamma 1$, Grb-2, Vav-1 and PDE-4A. Analysis of the supernatants of activated T cells with ligand-bound tumor vaccine showed that the vaccine/ bsHN-IL2, in contrast to the vaccine/bsHN-CD28, did not lead to the production of additional IL-2. We report here the first transcriptome analysis of IL-2 receptor mediated costimulatory signals. The findings provide new insights into mechanisms of function of IL-2 during T cell priming.
\end{abstract}

Correspondence to: Professor Volker Schirrmacher and Dr Philippe Fournier, German Cancer Research Center (DKFZ), Im Neuenheimer Feld 280, D-69120 Heidelberg, Germany

E-mail: v.schirrmacher@dkfz.de

E-mail: p.fournier@dkfz.de

Present address: ${ }^{1}$ Center of Immunology and Oncology (IOZK), Hohenstaufenring 30-32, 50674 Köln, Germany

Key words: bispecific antibodies, antitumor, vaccination, costimulation (signal 2), IL-2, signal 1 , T cell activation and proliferation, array, gene chip

\section{Introduction}

It is well accepted that the key players in protective anti-cancer adaptive immune responses are $\mathrm{T}$ lymphocytes. Patients with tumor-specific $\mathrm{T}$ cell responses, including those with $\mathrm{T}$ cell memory in their bone marrow (1) or those with tumor-infiltrating $\mathrm{T}$ lymphocytes (2) apparently have a better prognosis than those without such $\mathrm{T}$ cell immune responses. Nevertheless, the $\mathrm{T}$ cell responses are often not strong enough to prevent tumor growth. Various tumor inhibitory mechanisms account for this phenomenon, for instance impaired presentation of tumor-associated antigens (TAA) by MHC-I molecules $(3,4)$, the lack of costimulatory ligands for $\mathrm{T}$ cell activation (5) and the immunosuppressive microenvironment which a growing tumor is building up by secreting suppressive cytokines or by inducing regulatory $\mathrm{T}$ (Treg) cells that locally repress anti-tumor T cell attacks (6-8). Also, TAA-specific T cells may become anergized in cancer patients. There is thus a need for improved immunotherapeutic approaches to by-pass these problems.

Naive T cells, which represent about $50-80 \%$ of the total $\mathrm{T}$ cells, have more complex activation requirements compared to antigen experienced memory $\mathrm{T}$ cells. For their activation, the recognition of cognate antigen (e.g., MHC: peptide complex) by the TCR which provides signal 1 is not sufficient. Additional signals, called co-stimulatory signals, are required to induce proliferation and effector functions. Under physiological conditions, costimulatory signals are provided by professional antigen presenting cells such as DCs. B7 molecules (B7-1:CD80, B7-2:CD86), which interact with CD28 on T-cells, are the best-characterized costimulatory molecules $(9,10)$. Cross-linking of CD28 molecules on T cells in presence of signal 1 leads to the production of IL-2 (11-13), a cytokine pivotal for $\mathrm{T}$ cell growth, survival and cytotoxicity (14). CD28 ligation without cognitive interaction (in the absence of antigen recognition) has no effect on $\mathrm{T}$ cells. After activation, T-cells go from G0 into the G1 phase of the cell cycle. Simultaneously or successively, several surface molecules become upregulated, which either enhance or support the activation of effector functions. Activated T cells upregulate, among other molecules, LFA-1 (a heterodimeric integrin binding to ICAM-1 and ICAM-2), CD2 (a ligand for LFA-3) or VLA-4 ( $\alpha 4$ : $\beta 1$ integrin heterodimer, which binds to VCAM-1). Such adhesive molecular interactions strengthen the cognitive interactions between $\mathrm{T}$ cells and APCs and lead to improved migration and extravasation of 
$\mathrm{T}$ cells into inflammatory tissues $(15,16)$. Furthermore, the production of various cytokines is increased during $\mathrm{T}$ cell activation. For example, interleukin-2 (IL-2) and the $\alpha$ chain of the IL-2 receptor (IL-2R) are being induced. The latter consists of three chains: IL-2R $\alpha$ (CD25), IL-2Rß (CD122) and IL-2R $\gamma \mathrm{c}$ (CD132). Resting cells have a form of IL-2 receptor, which consists only of the $\beta$ - and $\gamma \mathrm{c}$-chain. These receptors have only a medium affinity for IL-2. Only through the formation of a trimer with the $\alpha$-chain, the receptor has a maximum affinity for its ligand IL-2 $(17,18)$. The IL-2 cytokine is important since it influences in an auto- and paracrine fashion the clonal expansion of T-cells (19).

In this study, we used the tumor selective replication properties of an avian paramyxovirus, the Newcastle Disease Virus (NDV) (20), to introduce at the surface of tumor cells the viral antigen Hemagglutinin-Neuraminidase (HN). This allows to target immunostimulatory molecules to such infected tumor cells. Human tumor cell infection by NDV was shown to be an efficient and safe way to produce the human cancer vaccine ATV-NDV (for autologous tumor vaccine infected by NDV). It showed pleiotropic immunostimulatory properties (21) and led to upregulation at the cell surface of the HN and F proteins of NDV (22).

As immunostimulatory molecules, we used 3 different recombinant proteins. The first, the bispecific single-chain antibody bsHN-CD3, provides $\mathrm{T}$ cell activation signals by interacting with the TCR-associated CD3 complex (signal 1). The second, the bispecific single-chain antibody bsHN-CD28 provides, via anti-CD28, costimulatory signals (signal 2). The third protein, the immunocytokine bsHN-IL2 introduces with IL-2 a tumor cell surface bound ligand for CD25 [interleukin-2 receptor (IL-2R)] on T cells. These molecules were introduced into the NDV infected vaccine to provide additional signals for $\mathrm{T}$ cell activation. Such additional signals may be required in cancer patients whose $\mathrm{T}$ cells exhibit a high degree of unresponsiveness to stimulation via TAA or in patients whose tumors do not express TAAs.

In this study, the effects of the fusion protein bsHN-IL2 of the modified vaccine have been analyzed in more detail. We analyzed the transcriptome of naive $\mathrm{T}$ cells after their activation, in the presence of suboptimal signal 1, with a tumor vaccine expressing IL-2 at their surface. We compared the stimulation of naive $\mathrm{T}$ cells by this vaccine to the one obtained with a vaccine providing a co-stimulatory signal via CD28. We also analysed the cytokine responses of such activated T cells. We will demonstrate that IL-2 immunocytokines, when targeted to NDV-infected tumor cells, and combined with a suboptimal TCR-mediated signal (signal 1; anti-CD3 as a surrogate for TAAs), provide a strong costimulatory signal to purified naive human $\mathrm{T}$ lymphocytes. Such IL-2 receptor-mediated costimulatory signals show many similarities but also some differences to 'classical' CD28-mediated costimulatory signals.

\section{Materials and methods}

Cell lines and preparation of the tumor vaccine. All cell lines were obtained from the tumor cell bank of the German Cancer Research Center (DKFZ; Heidelberg, Germany). Cell culture media were supplemented with $2 \mathrm{mM}$ L-glutamine,
$10 \mathrm{mM}$ HEPES, $100 \mathrm{U} / \mathrm{ml}$ penicillin and $100 \mu \mathrm{g} / \mathrm{ml}$ streptomycin (all purchased from Gibco Invitrogen, Karlsruhe, Germany). The human breast carcinoma cell line MCF-7 was cultured in DMEM with $10 \%$ fetal calf serum (FCS). The murine fibroblast Ltk-HN transfectant (23) and the Jurkat cell line sorted for CD28 expression (JCD28) was cultured in RPMI-1640 medium with 10\% FCS. Human peripheral blood mononuclear cells (PBMC) were isolated via Ficoll-Paque (Amersham Biosciences, Freiburg, Germany) centrifugation from buffy coat or from fresh heparinized blood of healthy donors. Untouched T cells (TCs) were purified with the T Cell Negative Isolation Kit (Dynal, Hamburg, Germany), involving magnetic separation of non-T cells labelled with mouse mAbs against human CD14, CD16a/b, CD56 and HLA-II DR/DP, via Dynabeads coated with human IgG4 against mouse IgG Fc. Naive T cells were purified in a similar way by adding an anti-CD45RO mAb to the above mentioned antibody cocktail to remove also memory $\mathrm{T}$ cells.

Tumor vaccine. For the preparation of the vaccine, the avirulent, non-lytic strain Ulster of NDV was used. Origin, maintenance, purification, and quantification have been described (21). MCF-7 cells were $\gamma$-irradiated via a ${ }^{137} \mathrm{Cs}$ source (Gammacell 1000; Atomic Energy of Canada, Chalk River, Canada), and then modified for $1 \mathrm{~h}$ at $37^{\circ} \mathrm{C}$ with 100 hemagglutination units (HU) of NDV per $1 \times 10^{7}$ cells (MCF-7NDV or V), as described (21).

Recombinant proteins. The bispecific antibodies bsHN-CD3 (no. 242) and bsHN-CD28 (no. 290) (24) as well as bsHN-IL2 (no. 356) (25) have been described. Briefly, the plasmid with the corresponding construct were elaborated using scFv specific for CD3, CD28 and HN of NDV derived respectively from the hybridoma clones TR66, 9.3 and HNB as desbribed in ref. 24. These plasmid were transfected in $\mathrm{CHO}$ cells and stable clones expressing high levels of the desired proteins were cultured in $\alpha$ MEM containing 5\% dialyzed FCS (dFCS; Biochrom, Krefeld, Germany) and $10 \mathrm{nM}$ methotrexate (Calbiochem, Schwalbach, Germany). The production was conducted in high density cell culture systems (Integra Biosciences, Fernwald, Germany). Each protein was then purified and characterized biochemically for our study. The protein concentration was determined with the CB-Protein Assay ${ }^{\mathrm{TM}}$ reagent (Calbiochem) and using bovine serum albumin (BSA, Sigma, Schnelldorf, Germany) as reference protein.

Antibodies and flow cytometry. PE-labelled goat $\mathrm{F}\left(\mathrm{ab}^{\prime}\right)_{2}$ antimouse IgG was from Southern Biotech (Birmingham, USA). PE-conjugated mAb against murine CD25 and the corresponding PE-conjugated rat IgG1 mAb as negative control were obtained from BD Pharmingen (Heidelberg, Germany). The PE-conjugated anti-CD3, the FITC-conjugated anti-CD4, the APC-conjugated anti-CD25 as well as the FITC-conjugated anti-CD45RA mAbs were purchased from BD Pharmingen. The anti-EpCAM mAb (HEA-125) was a kind gift of Dr G. Moldenhauer (DKFZ). The purified anti-CD45RO, the allophycocyanin (APC)-conjugated anti-CD8, the fluorescein isothiocyanate (FITC)-conjugated anti-CD25 as well as the phycoerythrin (PE)-labelled anti-CD45RO and anti-CD107a 
mAbs were purchased from Becton-Dickinson (BD) Pharmingen. The secondary antibodies goat $\mathrm{F}\left(\mathrm{ab}^{\prime}\right)_{2}$ anti-mouse PE Mouse IgM, IgG, IgA $(\mathrm{H}+\mathrm{L})$ and the goat anti-mouse FITC mouse $\mathrm{IgG} 2 \mathrm{a}$ (only $\mathrm{H} \gamma 2 \mathrm{a}$ ) were purchased from Southern Biotech, Birmingham (USA) whereas Endobulin (human, intact IgG) from Baxter (Unterschleissheim, Germany) and streptavidin-PE from BD Pharmingen.

For the analysis of human naive $\mathrm{T}$ cell activation, $\leq 1 \times 10^{6}$ $\mathrm{T}$ lymphocytes were first blocked with Endobulin (human immunoglobulins, $50 \mu \mathrm{g} / \mu 1$; Baxter, Unterschleissheim, Germany), before being stained for flow cytometry. The cell suspensions were analyzed with a FACSCalibur fluorescence analyzer (Becton-Dickinson). In some proliferation assays, $\mathrm{T}$ cells were labelled by short incubation with a CFSE solution. Propidium iodide (PI, $0.5 \mu \mathrm{g} / \mu \mathrm{l}$; Sigma-Aldrich, Schnelldorf, Germany) was added to all cell suspensions immediately before the FACS analysis to exclude dead cells. The data were analyzed using the FlowJo software (Tree Star, Ashland, USA).

Mixed lymphocyte tumor cell culture (MLTC). In this assay, $1 \times 10^{5}$ freshly isolated naive $\mathrm{T}$ cells per well were co-incubated in 96-well round bottom microtiter plates with $1 \times 10^{4}$ stimulator cells (Ltk-HN cells). After the indicated durations of incubation in presence or absence of the fusion proteins, the cells were harvested, washed and either used in the TNA assay, for array analysis (described below) or stained for FACS analysis (see above).

Tumor neutralization assay (TNA). The assay system was based on (24) with some minor modifications. Briefly, using 96-well plates, $3 \times 10^{3}$ live MCF-7 tumor cells were added to the wells and incubated in culture medium for $4 \mathrm{~h}$ until they became adherent. Purified naive T cells that had been preactivated in an MLTC were added to the wells at an effector to tumor cell ratio of 5:1. Then, the indicated amounts of the recombinant proteins were added and incubated until the tumor monolayer in the control wells was confluent.

At the end of the assay, the supernatants of the 96-well TNAs were removed and the wells were carefully washed 5 times with $200 \mu 1$ RPMI-1640 medium (plus 5\% FCS) to remove non-adherent cells. After the last wash, the wells were filled with $100 \mu 1$ RPMI-1640 medium (plus 5\% FCS) and $20 \mu \mathrm{l}$ MTS solution (MTS Cell Titer 96 ${ }^{\circledR}$ AQueous; Promega, Mannheim, Germany) was added. The plates were then incubated for $45 \mathrm{~min}$ at $37^{\circ} \mathrm{C}$ in the dark. When, in the control [tumor monolayer wells [without MLTC)], the colour switched from yellow to brown, the reaction was stopped by adding $20 \mu 110 \%$ SDS per well. The plates were then evaluated in an ELISA reader at $490 \mathrm{~nm}$. The background was taken from 3 control wells containing only medium, MTS reagent and SDS solution. The percent tumor growth inhibition was calculated as described (24).

Total RNA extraction and synthesis of labelled cRNA. All kits were purchased from SuperArray (Frederick, USA). After activation of naive $\mathrm{T}$ cells for seven days, potentially remaining MCF-7-NDV vaccine cells were removed from the co-cultures by magnetic beads (Dynal, Hamburg, Germany) pre-loaded with an anti-EpCAM mAb. The remaining T cells were lysed and total RNA extracted with the ArrayGrade ${ }^{\mathrm{TM}}$ Total RNA
Isolation Kit (SuperArray). RNA from freshly isolated naive $\mathrm{T}$ cells of the same donor served as reference. Using the TrueLabeling-AMP ${ }^{\mathrm{TM}}$ Linear RNA Amplification Kit (SuperArray), the RNA samples were first reverse transcribed into cDNA and then amplified linearly by in vitro transcription into cRNA, a step during which the cRNA was labelled with biotin-16-uridine-5'-triphosphate (biotin-16-UTP, $10 \mathrm{mM}$; Roche, Mannheim, Germany). Finally, the cRNA samples were purified with the use of the ArrayGrade cRNA Cleanup Kit (SuperArray).

Hybridization onto oligo microarrays and image acquisition. Gene expression analysis involved the use of customized Oligo DNA Microarrays (SuperArray) containing 247 different human gene probes potentially involved in $\mathrm{T}$ cell activation (supplemental data available on request from the authors). Ten microgram of each biotinylated cRNA sample was hybridized overnight at $60^{\circ} \mathrm{C}$ to the microarrays, following the manufacturer's instructions (SuperArray). The signals were developed with streptavidin-conjugated alkaline phosphatase and the CDP substrate (Chemiluminescent Detection; SuperArray). The detection was performed with the aid of a cooled CCD camera (Roche, Mannheim, Germany) during an exposure time of $10 \mathrm{~min}$. The images were saved as TIFFformatted 16 bit gray scale images. Data analysis, i.e. quantification of the signal intensities, background correction and normalization with the ribosomal protein S27a (RPS27A) as a house-keeping gene, was carried out with the use of the GEArray Expression Analysis Suite software (v. 1.1; SuperArray). The relative gene expression is given as fold induction in comparison to the reference sample (set as 100\%).

Cytokine measurement. The Th1/Th2 response-related cytokines IFN- $\gamma$, IL-1ß, IL-2, IL-4, IL-5, IL-6, IL-8, IL-10, TNF- $\alpha$ and TNF- $\beta$ from supernatants were measured simultaneously by means of a commercially available bead-assisted FACS analysis (Bender MedSystems, Vienna, Austria). IP-10 was quantified by a commercial ELISA (Biosource, Camarillo, USA).

Statistical analysis. Differences between two means were calculated in Excel software with the Student's t-test. They were regarded as statistically significant at $p<0.05$.

\section{Results}

Binding properties of the recombinant proteins $b \mathrm{sH}-\mathrm{CD} 3$, bsHN-IL2 and bsHN-CD28. Fig. 1 shows a scheme of the recombinant fusion proteins and how they function in combination with virus infected tumor cells to activate co-cultured $\mathrm{T}$ cells. The 3 recombinant fusion proteins bsHN-CD3 (no. 242), bsHN-CD28 (no. 290) and bsHN-IL2 (no. 356) were first characterized for their binding to human $\mathrm{T}$ cells. To this end, purified T cells (TC) were activated for $48 \mathrm{~h}$ with autologous dendritic cells (DC) in the presence of the bacterial superantigen SEB in a TC/DC ratio of 5:1. This co-culture led to the expression of CD25 [the $\alpha$-subunit of the heterotrimeric IL-2 receptor (IL2R)] on their surface (data not shown). The use of $\mathrm{CD} 3 / \mathrm{CD} 28$ expander beads has been circumvented in order not to occupy the binding sites for the fusion proteins. 


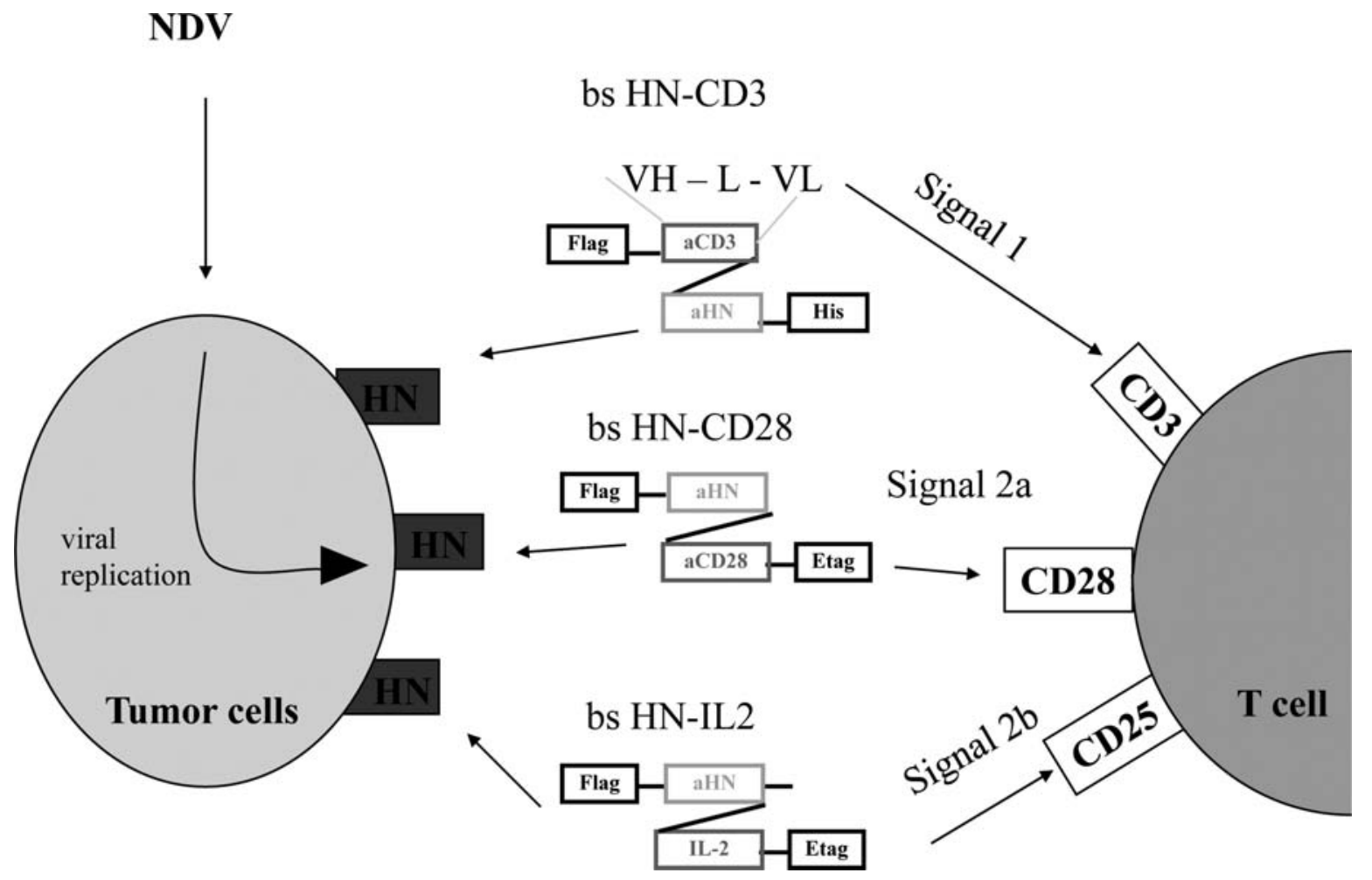

Figure 1. Activation of T cells, in the presence of signal 1, by membrane-bound ligands [bsHN-CD28 (no. 290) and bsHN-IL2 (no. 356)] bound to the surface of stimulator cells via the viral HN protein introduced upon NDV infection. For the elaboration of the ATV-NDV tumor vaccine, tumor cells are irradiated and infected with NDV. This leads through viral replication to the expression of the viral HN protein at the cell surface. The latter is used as anchor for the coupling of three different proteins. The bsAb bsHN-CD3 (no. 242) is a bispecific antibody binding to HN via one arm and to CD3 via the other arm. It introduces, by binding to $\mathrm{HN}$ expressed at the cell surface of the tumor vaccine, the signal 1. BsHN-CD28 (no. 290) is a bispecific protein binding to HN and to CD28. Similarly to the former protein, it confers the signal 2 a to the vaccine. The fusion protein bsHN-IL2 (no. 356) binds to HN and thereby presents IL-2 activity as CD25 ligand at the surface of the tumor vaccine. In this study, we studied the triggering of naive T cells via CD25 presented at the surface of the tumor vaccine using the IL-2 fusion protein in the presence of suboptimal signal 1 and compared it to the one induced via CD28 by bsHN-CD28 bound to the tumor vaccine.

As shown in Fig. 2Aa, non-activated T cells form a very compact, homogeneous cell population (long arrow) with small FSC and SSC values whereas the SEB activated T cells (small arrows) differentiated into lymphoblasts, which have a wide variation for both parameters (Fig. 2Aa). Both proteins, bsHN-CD3 and bsHN-CD28, bound to their respective receptors (CD3 and $\mathrm{CD} 28$ ) on the preactivated T cells as shown by the histograms of Fig. 2Ab and c. BsHN-IL2 also bound to the $\mathrm{T}$ cells but there were two binding peaks (Fig. 2Ad). The middle one corresponds to low affinity binding (CD25 low $\mathrm{T}$ cells) and the right to high affinity binding (CD25 high $\mathrm{T}$ cells). The expression level of the IL $2 \mathrm{R} \alpha$ thus appears to correlate with the degree of $\mathrm{T}$ cell activation and with binding of bsHN-IL-2.

Binding of the constructs to $\mathrm{CD} 28$ or to the IL-2 receptor was also tested with Jurkat (JCD28) CD28 positive human lymphoma cells and with IL-2 receptor positive murine $\mathrm{Eb}$ lymphoma cells, respectively. The cells were first incubated with the recombinant proteins and then stained with mouse anti-His-tag or mouse anti-E-tag followed by PE-labelled goat $\mathrm{F}\left(\mathrm{ab}^{\prime}\right)_{2}$ anti-mouse IgG and then analyzed by flow cytometry (data not shown). To test binding to HN, the proteins were successively diluted 1:2 and their binding to Ltk-HN transfectants analyzed. The dilution, at which $50 \%$ of the cells were stained, was set as arbitrary value of $10,000 \% / \mathrm{ml}$ $(\mathrm{U} / \mathrm{ml}) \mathrm{HN}$-associated activity. The protein bsHN-CD28 (no. 290) showed a binding activity of about $500,000 \mathrm{U} / \mathrm{ml}$ which is 2.5-times higher than the activity observed for the protein no. 356 (with $200,000 \mathrm{U} / \mathrm{ml}$ ). Table I shows the HN specific activity in terms of $\mathrm{U} / \mu \mathrm{g}$ protein and also the IL-2 specific activity.

Phenotypic characterisation of the isolated human naive $T$ cells. The naive $\mathrm{T}$ cells which have been freshly isolated and purified were stained directly with fluorescence labelled antibodies for FACS analysis. As shown in Fig. 2Ba, all cells were CD3 and CD45RA positive. They did not express the memory T-cell marker CD45RO, or CD25 (Fig. 2Bd and e), nor CD107a (Fig. 2Bf). Regulatory T cells (Treg) were also not present.

Bystander anti-tumor activity induced in naive T cells by IL-2 expressed at the surface of tumor vaccines. We next evaluated possible bystander anti-tumor activity of $\mathrm{T}$ cells activated via IL-2 expressed at the surface of a tumor vaccine. For this purpose, we used the tumor-neutralization assay (TNA) which is basically a mixed lymphocyte tumor cell culture (MLTC) performed on top of an adherent tumor cell monolayer as bystander target (24). Co-incubation of PBMC for 5-7 days with the modified vaccine cells resulted in the generation of cytotoxic effector function and in the release of a variety of cytokines. During this effector phase, the bystander tumor cells were lysed or their proliferation inhibited. At the end of the assay, the amount of surviving tumor cells was measured 
A
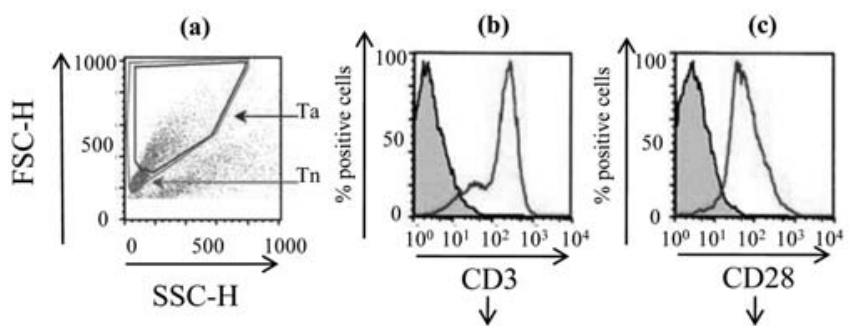

(d)

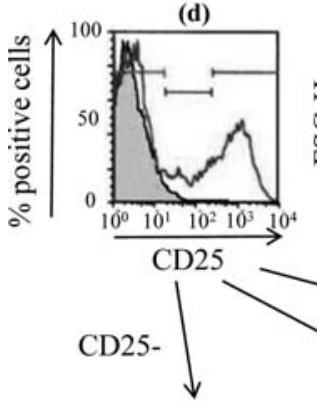

(g)
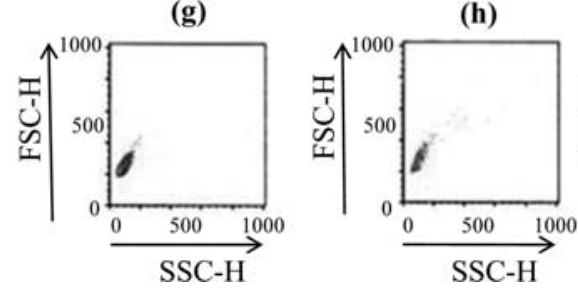

(h)

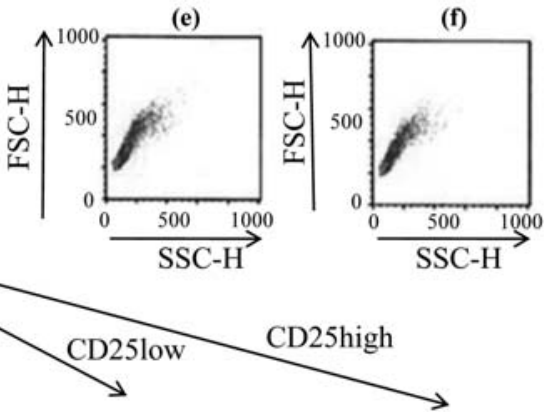

(i)

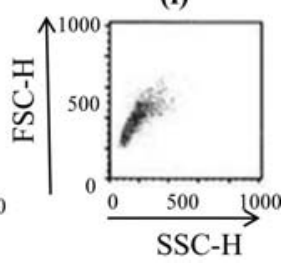

B

(a)

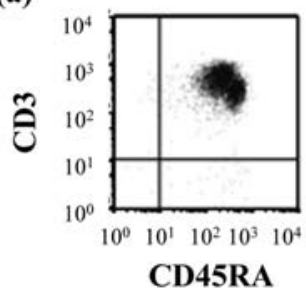

(b)

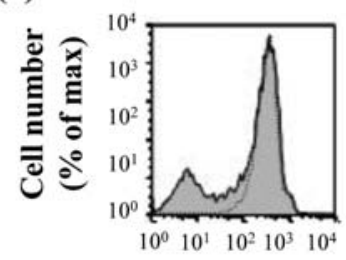

CD45RA

(d)

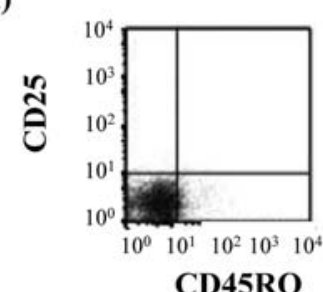

(f)

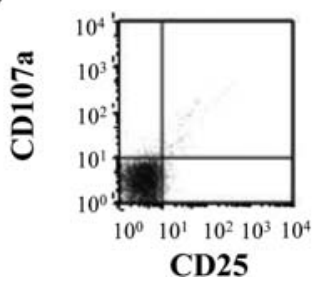

(c)

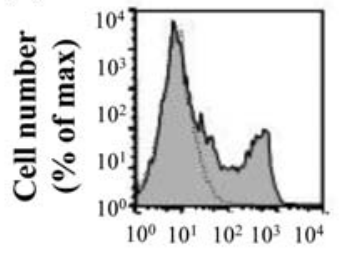

CD45RO

(e)

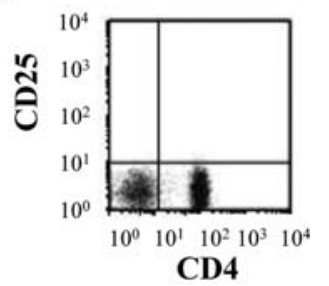

(g)

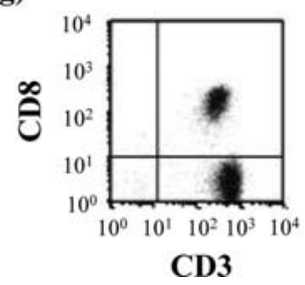

C

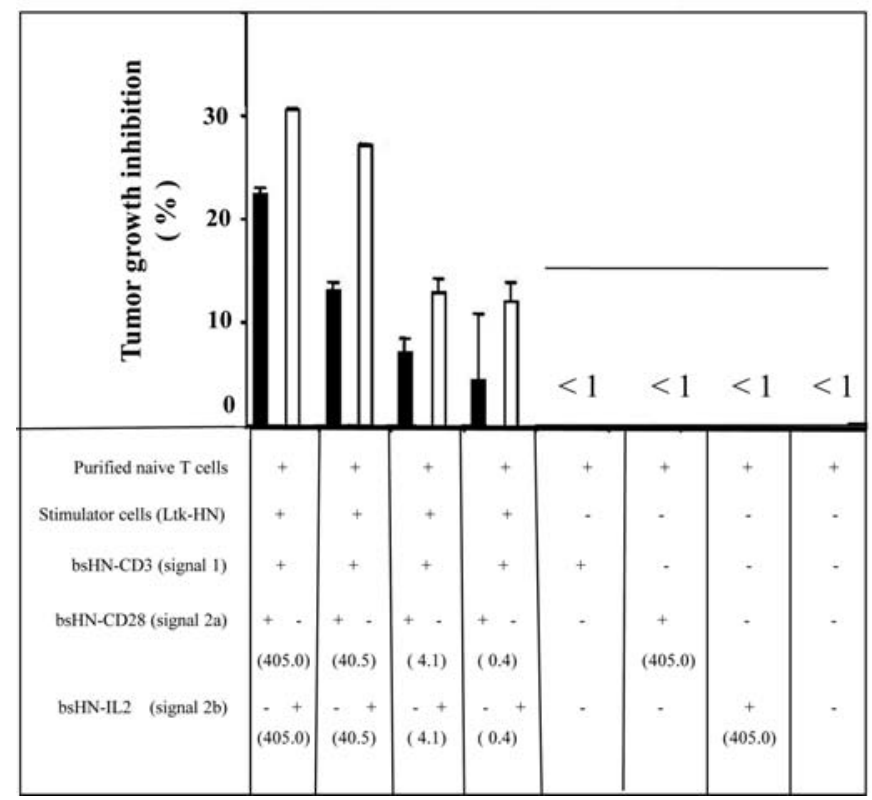

Figure 2. Binding of the recombinant proteins bsHN-IL2 (no. 356) and bsHN-CD28 (no. 290) to T cells, characterisation of purified naive T cells and increased bystander antitumor activity induced by NDV tumor vaccine in naive T cells through addition of both proteins. (A) Binding of bsHN-CD3 (no. 242), bsHN-CD28 (no. 290) and bsHN-IL2 (no. 356) to T cells. Purified $\mathrm{T}$ cells (TC) were activated for $48 \mathrm{~h}$ in the presence of autologous dendritic cells (DC) and SEB $(1 \mathrm{~g} / \mathrm{ml})$ at a TC/DC ratio of 5:1. A dot plot FSC-H vs SSC-H representation shows a difference of size and granularity between activated and non-activated T cells (a): The black arrows highlights the activated $\mathrm{T}$ cells while the grey arrows show the localization of the not activated T cells. For FACS staining, 5-7 $\times 10^{5} \mathrm{TC}$ were first incubated with 12.5-51.7 $\mu \mathrm{g}$ of the fusion proteins no. 242 (b), no. 290 (c) and no. 356 (d). TC were then stained with anti-His (b and d) or anti-E-tag (c) primary antibody and then with the PE-conjugated goat anti-mouse IgG. For the negative control, samples were only incubated with an isotype control mAb added at the same concentration as the primary antibody and then with the secondary antibody (grey curve). The size and granularity of $\mathrm{CD}^{+}$cells (e) and $\mathrm{CD} 28^{+}$cells were analyzed. In a similar fashion, a dot plot $\mathrm{FSC}-\mathrm{H}$ vs SSC-H representation has been drawn for the subpopulations CD25- (g), CD25low (h) and CD25high (i). Dead cells were excluded of the measurement by PI staining. (B) Characterization of the purified human naive $\mathrm{T}$ cells by flow cytometry. Naive $\mathrm{T}$ cells were isolated as described in Materials and methods, stained with fluorescence-labelled mAbs and analyzed by flow cytometry. (a) Purified naive $\mathrm{T}$ cells were double stained with FITC-labelled anti-CD45RA and PElabelled anti-CD3 mAbs; (b and c) Purified naive T cells (dotted lines) were stained with FITC-labelled anti-CD45RA (left histogram overlay (b) or with PE-labelled anti-CD45RO (right histogram overlay (c). The shaded histograms represent total $\mathrm{T}$ cells isolated as described in Materials and methods without the use of the anti-CD45RO mAb to remove the memory $\mathrm{T}$ cell fraction. (d) Purified naive T cells were double stained with PE-labelled antiCD45RO and APC-labelled anti-CD25 mAbs. (e) Purified naive T cells were double stained with FITC-labelled anti-CD4 and APC-labelled anti-CD25 mAbs. Note that $\mathrm{CD}_{4}^{+} \mathrm{CD} 25^{+} \mathrm{T}$ regulatory cells are absent from naive $\mathrm{T}$ cell preparation. (f) Purified naive T cells were double stained with FITC-labelled anti-CD25 and PE-labelled anti-CD107a mAbs. (g) Purified naive T cells were double stained with PE-labelled anti-CD3 and APC-labelled anti-CD8 mAbs. (e and g) Purified naive T cells contain both $\mathrm{CD}^{+}$helper and $\mathrm{CD} 8^{+}$ cytotoxic $\mathrm{T}$ cells. (C) Bystander anti-tumor activity induced in naive $\mathrm{T}$ cells through bsHN-IL2 in the presence of suboptimal signal 1. In a 96-well plate, purified naive $\mathrm{T}$ cells $\left(1 \times 10^{5}\right)$ were activated by co-incubation with $1 \times 10^{4}$ irradiated Ltk-HN cells, suboptimal bsHN-CD3 (50 ng/well) and the reported amounts of bsHN-CD28 (black bars) or bsHN-IL2 (white bars). Controls were naive $\mathrm{T}$ cells which were incubated only with the vaccine or with the vaccine to which one of the constructs is bound. After six days, the cells were harvested and given to one in a 96-well plate in which MCF-7 cells $\left(2.5 \times 10^{4}\right.$ cells/well) were added $4 \mathrm{~h}$ beforehand. After three days of incubation, the tumor viability was quantified as described in Materials and methods. All values were determined in triplicate and are expressed as mean \pm standard deviation. 
Table I. Summary of the characteristics of the 2 purified recombinant proteins bsHN-IL2 and bsHN-CD28 used in this study.

\begin{tabular}{lccc}
\hline $\begin{array}{l}\text { Protein } \\
\text { no. }^{\text {a }}\end{array}$ & $\begin{array}{c}\text { Protein } \\
\text { structure }^{\mathrm{a}}\end{array}$ & $\begin{array}{c}\text { HN specific } \\
\text { activity }^{\mathrm{c}} \\
(\mathrm{U} / \mu \mathrm{g})\end{array}$ & $\begin{array}{c}\text { IL-2 specific } \\
\text { activity }^{\mathrm{d}} \\
(\mathrm{U} / \mu \mathrm{g})\end{array}$ \\
\hline 290 & $\begin{array}{c}\text { bsHN-CD28 } \\
\text { bsHN-IL2 }\end{array}$ & $\begin{array}{c}0.204 \times 10^{5} \\
0.019 \times 10^{5}\end{array}$ & $0.0135 \times 10^{6}$ \\
\hline
\end{tabular}

aThe protein number refers to the plasmid that codes for the particular protein, the clone number to the well of the 96-well plate from where the clone was isolated. ${ }^{b}$ The 2 recombinant proteins contained specific binding activities for the viral HN surface antigen and for CD3 (not shown) and CD28 (bottom) as single-chains antibodies. The N- and C-terminal Tag sequences, such as Flag tag or His6 were used for purification as well as for detection of the recombinant proteins in Western blot or FACS. The two recombinant proteins are characterized for their specific $\mathrm{HN}$-activity by determinate their titer in flow cytometry. The $\mathrm{HN}$-activity was quantified by titrating the proteins on $\mathrm{HN}$-expressing Ltk mouse fibroblasts followed by FACS analysis. An arbitrary activity of $10,000 \mathrm{U} / \mathrm{ml}$ was attributed to a protein dilution which stained $50 \%$ of the Ltk-HN cells. ${ }^{\mathrm{c}}$ After determination of their protein concentration by Bradford, a specific activity was calculated. ${ }^{\mathrm{d}}$ Their IL-2 activity was assessed by using the IL-2 dependent cell line CTLL). The plasmids encoding the bispecific constructs bsHN-CD3 (no. 242) and bsHN-CD28 (no. 290) (24), as well as bsHN-IL-2 (no. 356) (25) have been described previously. Briefly, transfection of the plasmids into $\mathrm{CHO}$ cells was carried out by electroporation (Bio-Rad, Munich, Germany). Clones that were stably expressing the desired fusion protein were selected by limiting dilution technique. The production was conducted in high density cell culture systems (Integra Biosciences, Fernwald, Germany). The fusion proteins containing a His6-tag at their C-terminus were purified by immobilized metal affinity chromatography (IMAC), as described (24). The E-tag containing protein (bsF-CD28) was purified by means of an anti-E-tag immunoaffinity chromatography procedure (Amersham Biosciences, Freiburg, Germany). The purity of the different fractions was assessed by Coomassie staining after separation of the purified proteins on $12.5 \%$ SDS-PAGE gels. The size of the molecules was checked by Western blotting. The fractions exhibiting the highest purity and protein amount were pooled, dialyzed against PBS, filtered $(0.22 \mu \mathrm{m})$ and stored at $-80^{\circ} \mathrm{C}$.

after removal of the non-adherent cells by addition of MTS, a live cell staining reagent. Strong bystander tumor growth inhibition was observed when a suboptimal anti-CD3 signal was combined with bsHN-IL2 or bsHN-CD28 at the surface of the tumor vaccine (data not shown).

Since the TNA assay does not allow to distinguish between steps of $\mathrm{T}$ cell activation and effector function, we next tested the generated effector function separately. MLTC cultures were first performed with naive T cells in the absence of tumor monolayers for six days with Ltk-HN stimulator cells and the indicated $\mathrm{HN}$-specific fusion proteins. Upon transfer onto MCF-7 human breast carcinoma cells, the generated effector $\mathrm{T}$ cells exerted within three days the tumor growth inhibition shown in Fig. 2C. There was a significantly higher effector activity in cultures in which a costimulus was provided
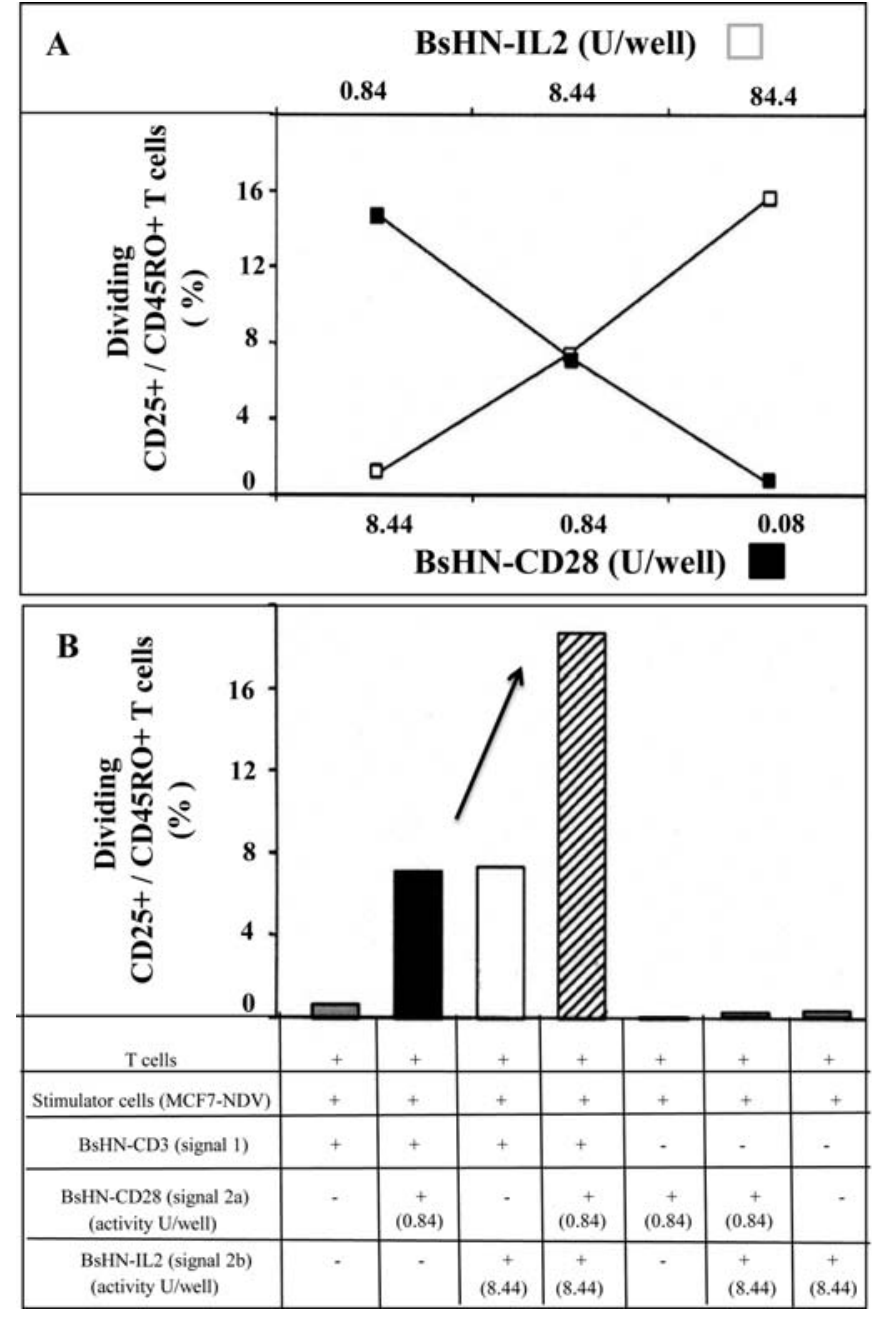

Figure 3. Activation and proliferation of naive $\mathrm{T}$ cells by a combination of both proteins bsHN-CD28 and bsHN-IL2. Purified naive T cells were labeled with CFSE. They were then incubated with $1 \times 10^{4}$ irradiated MCF-7 cells which were modified with $100 \mathrm{HU}$ NDV Ulster/107 cells, suboptimal bsHN-CD3 $(500 \mathrm{pg} / \mathrm{well})$ and three different amounts of bsHN-CD28 (8.44, 0.84 and $0.084 \mathrm{U} /$ well) or bsHN-IL2 $(84.4,8.44$ and $0.84 \mathrm{U} /$ well). Naive T cells only with the vaccine or in contact with one of the recombinant proteins served as controls. After six days, the cells were harvested, blocked with Endobulin and stained with anti-CD25-APC and anti-CD45RO-PE before being analyzed by flow cytometry. Dead cells were excluded by PI staining. The figure reflects the percentage of dividing CD25 and CD45RO co-expressing naive T cells of the total number of cells. (A) Titration of the costimulatory proteins in the presence of suboptimal concentrations of bsHN-CD3. (B) Combination of the recombinant proteins bsHN-CD28 and bsHN-IL2 at their respective suboptimal concentrations as defined in (A).

(either via $\mathrm{CD} 28$ or via $\mathrm{CD} 25$ ), in the presence of a suboptimal signal 1. The costimulation via CD25 induced an even stronger bystander anti-tumor effect than the costimulation via anti-CD28.

Synergism and cross-talk between costimulatory signals mediated via IL-2 receptor $(C D 25)$ and $C D 28$. To test the relationship between the co-stimulatory signals mediated via CD25 or CD28, we labelled purified naive T cells with CFSE and co-incubated them for 6 days with a tumor vaccine consisting of irradiated and NDV-infected human MCF-7 breast carcinoma cells. All experimental groups then received a suboptimal amount of bsHN-CD3 alone and 3 different doses 
A

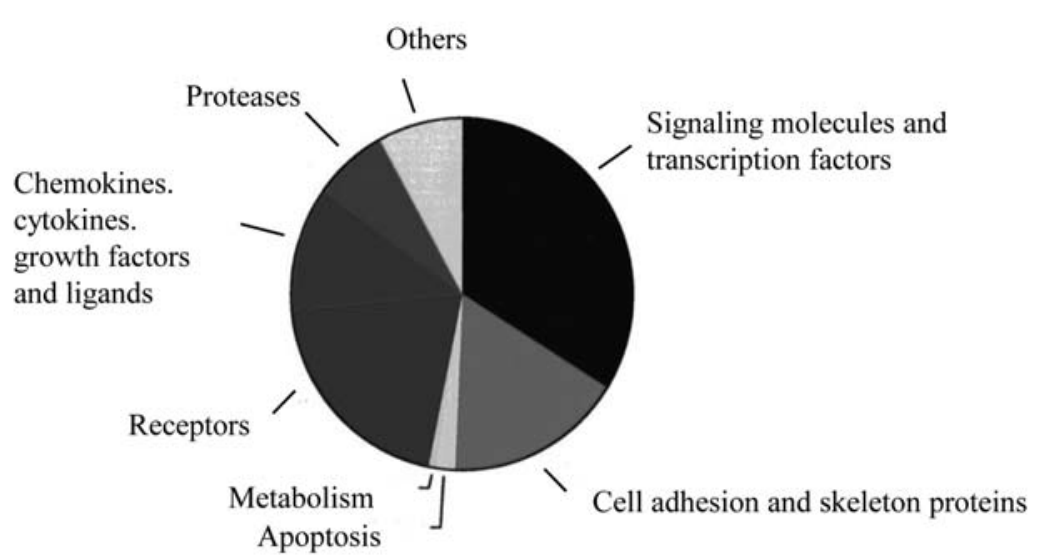

B

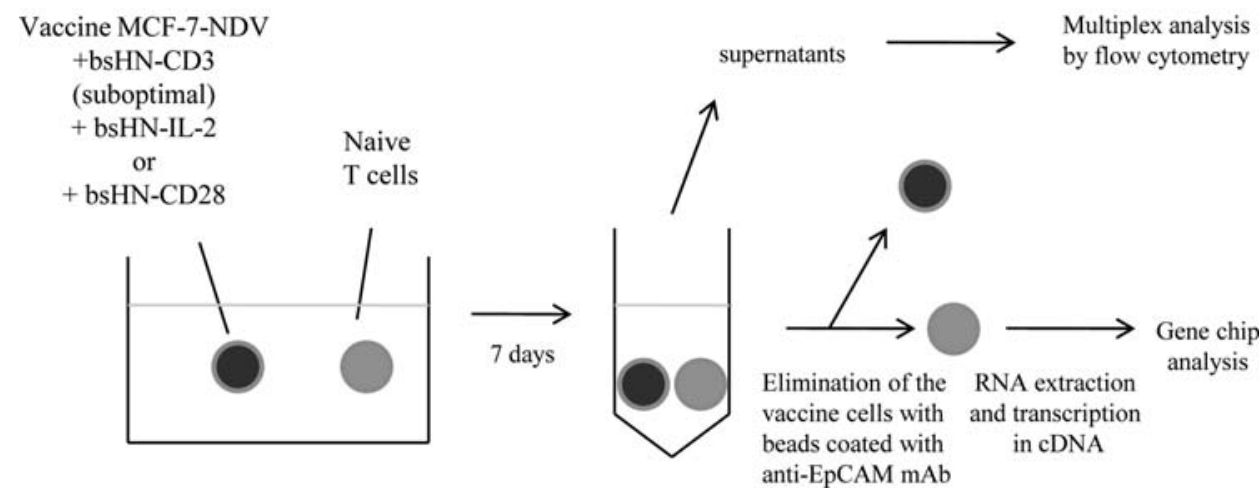

Figure 4. Set up for gene expression analysis for comparison between bsHN-IL2 and bsHN-CD28 for the triggering of naive T cells (in the presence of suboptimal signal 1). (A) Percentage distribution of the 247 genes on the gene chip. To enable an accurate analysis of gene expression, a cooperation was set up with the company SuperArray for the development of a gene chip, which contained a total for 247 gene-specific oligonucleotide probes. The genes, which are involved in T-cell activation included the following seven families of genes: signaling molecules and transcription factors, cell adhesion and cytoskeleton, apoptosis regulators, energy metabolism, receptors, a group of chemokines, cytokines, growth factors and ligands and finally proteases. Genes that could not be clearly assigned to these families, was grouped under the name 'others'. A complete list of the genes together with annotations can be found in the annex. (B) Schema representing the procedure followed to analyze the transcriptome of naive T cells after stimulation with the bsHN-IL2 or bsHN-CD28 loaded MCF7 tumor vaccine. Naive T cells were isolated from $500 \mathrm{ml}$ of fresh blood from a healthy donor and their nearly $100 \%$ purity confirmed by FACS (see Fig. 2). The activation was performed in 96-well plates. As vaccine were used MCF-7 cells which were irradiated and modified with $100 \mathrm{HU}$ NDV and which were loaded with suboptimal bsHN-CD3 (500 pg/well) in the presence of co-stimulatory bsHN-CD28 or bsHN-IL2 (each 84.4 U/well) or only of one of the three constructs. Unloaded vaccine cells served as controls. $1 \times 10^{7}$ freshly isolated naive T cells were added. A part of non-activated T cells was used to purify RNA for the determination of the zero value (24). The activation cultures were performed over 7 days, at the end of which microscopic differences were visible. The cells were then centrifuged at $250 \mathrm{~g}$, the supernatants carefully removed and stored until their use at $-80^{\circ} \mathrm{C}$. In order to avoid potential interference by possibly remaining vaccine cells, the cells mixture were incubated with magnetic particle-mouse IgG, which were coated with the anti-EpCAM mAb (HEA-125). The obtained T cells were lysed and total RNA isolated. The RNA samples were then transcribed into cDNA, which served as a template for the subsequent cRNA synthesis. In the last step, the cRNA was amplified and simultaneously marked by the use of biotin-16-UTP. Before the cRNAs could be used on the gene chip, it was purified on an affinity column. The amounts of RNA were thus quantified and were found to be between 33.8 and $46.2 \mu \mathrm{g}$. The units, which were determined photometrically as OD260/OD280 ratio, were observed for all samples to be >2,280 (data not shown). Ten micrograms cRNA of each sample were hybridized overnight at $60^{\circ} \mathrm{C}$ on an array according to manufacturer's instructions. The signals during the chemiluminescence reaction were analyzed with an alkaline phosphatase-conjugated streptavidin, the CDP-substrate and a CCD camera with an exposure time of 10 min. The images were stored as 16 -bit greyscale images in TIFF format. The analysis of the data, i.e. quantification of the signals, their correction and normalization using the ribosomal protein S27a (RPS27A) gene as house-keeping gene, was carried out automatically by the software GEArray Expression Analysis Suite. All signals were also checked again manually.

of bsHN-CD28 or bsHN-IL2 in all possible combinations. We then determined by FACS analysis the percentage of viable dividing cells (cells with reduced CFSE) co-expressing CD25 and CD45RO. Each of the two co-stimuli alone showed dose-dependent $\mathrm{T}$ cell activation over three log scales (Fig. 3A). Fig. 3B shows a synergistic effect (see the arrow) when CD25 and CD28 mediated signals were combined in suboptimal concentrations. The data suggest that deficiencies of anti-CD28 signals can be compensated by IL- 2 and vice versa.
Co-stimulatory signalling pathways elucidated via gene expression profiling. Next we performed a transcriptome analysis to compare the signaling molecules induced by the stimulus bsHN-CD28 (signal 2a) and by the stimulus bsHN-IL2 (signal 2b). Specially designed gene chips were loaded with oligonucleotides specific for 247 genes involved in $\mathrm{T}$ cell activation (Fig. 4A). Naive $\mathrm{T}$ cells were isolated from $500 \mathrm{ml}$ fresh blood and their purity evaluated by FACS analysis $(\sim 100 \%)$. The activation was performed in 96-well 
(a)

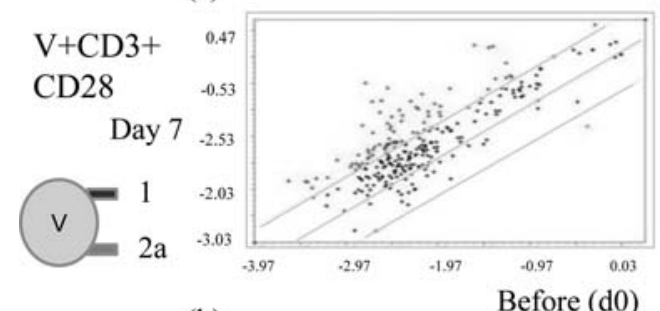

(b)

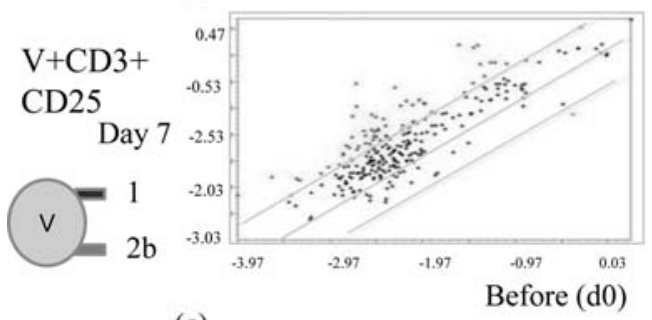

(c)

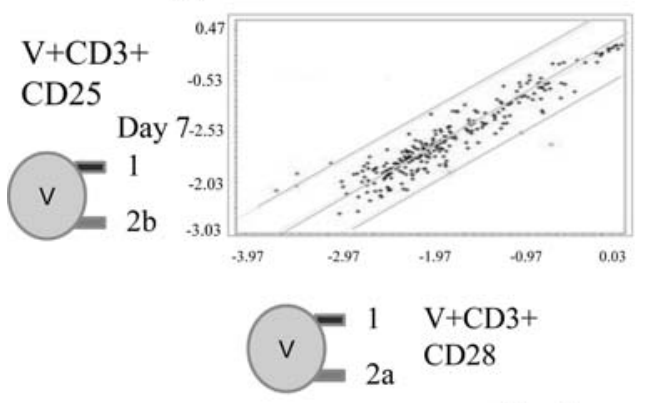

Day 7 (d)
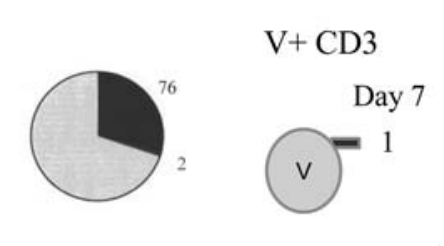

(e)
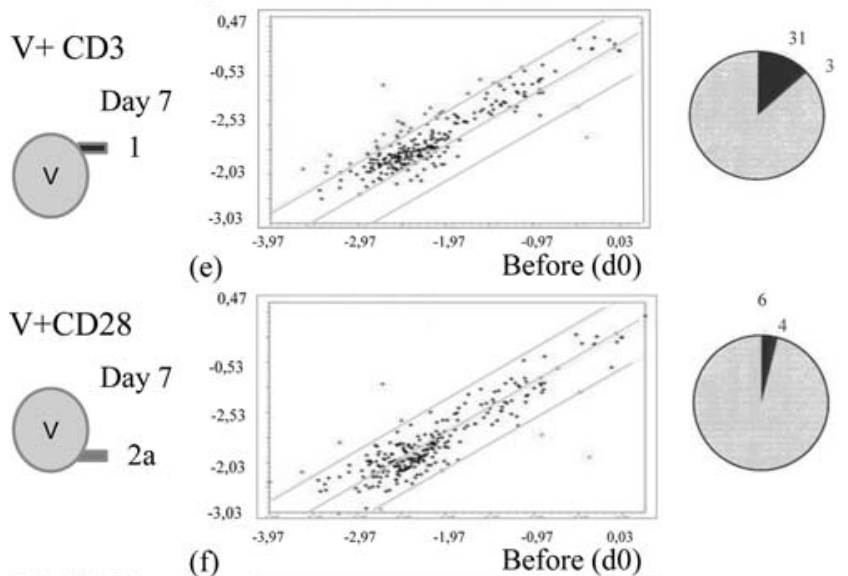

(f)
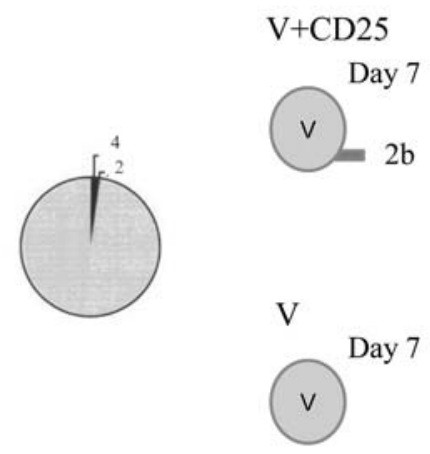

(g)
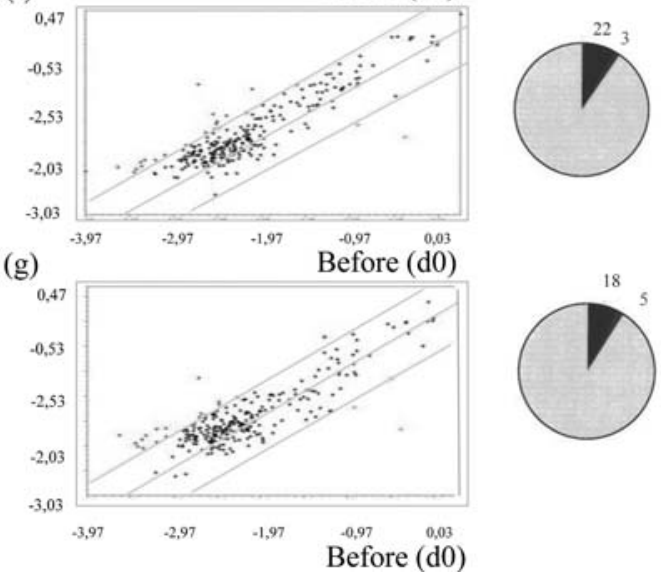

Figure 5. Gene expression profiling of naive T cells activated by IL-2 and anti-CD28 presented at the surface of the tumor vaccine. The results of gene chip analysis are represented as scatter plots (left) and pie charts (right). Naive T cells were stimulated with the 7 types of vaccine indicated. A total of seven samples (S1-S7) were analyzed. The total RNA of the reference (zero value) was obtained on day 0 (d0) from unstimulated 1x107 purified naïve T cells. The activation was carried out in 96-well plates. Naïve T cells were incubated with 1x104 MCF-7 cells which were irradiated and modified with NDV (100 HU/ 10 up to 7 cells). Suboptimal amounts of bsHN-CD3 (500 pg/well) and 84.4 U/well of bsHN-CD28 or bsHN-IL2 were added. Naïve T cells without the vaccine, with only the vaccine, and with one of the proteins served as controls (as mentioned in this table). After seven days of activation (d7), total RNA of the cells was isolated. The samples were compared to the reference gene expression of naive $\mathrm{T}$ cells at day 0 without activation. The different vaccines used for T-cell activation were (a) V+CD3+CD28; (b) V+CD3+CD25; (d) V+CD3; (e) V+CD28; (f) V+CD25; and (g) V. In the scatter plots of (c) the two costimuli CD28 and IL-2 are compared to each other in the presence of suboptimal signal 1.

plates with the respective vaccine and, after 7 days, RNAs were prepared from the T cells. All RNA samples exhibited high purity, as assessed by measurement of the optical density (OD) at 260 and $280 \mathrm{~nm}$, respectively. It turned out that the amount of RNA from fully activated naive T cells was three to four times greater than that of the controls.

After reverse transcription, the cDNAs were hybridized and analyzed as described in Materials and methods. In T cells activated via anti-CD3 and IL-2 (CD3/IL-2), 71 genes became upregulated (Fig. 5Ab) while in cells activated via anti-CD3 and anti-CD28 (CD3/CD28), 76 genes became upregulated (Fig. 5Aa). In both stimulation cultures, two genes (CXCR4 and the transcription factor Fos) became downregulated. These genes, however, were also downregulated in control cultures indicating an effect of the cell culture as such (Fig. 5Ag). The suboptimal anti-CD3 stimulus alone upregulated 18 genes (Fig. 5Ad), the anti-CD28 stimulus alone 6 genes (Fig. 5Ae) and IL-2 alone 22 genes (Fig. 5Af). In T cells stimulated only by the virus-modified tumor vaccine, there were 18 genes upregulated (Fig. 5Ag). We conclude that in these control cultures, the upregulation was usually less strong than in the fully activated cultures. Fig. 6 shows quantitative results for some selected genes. A comparison of the black bars reveals that many of the 23 selected upregulated genes were similarly affected by stimulus $2 \mathrm{~b}$ and stimulus $2 \mathrm{a}$. Since the signals are induced via different receptors, namely CD25(IL-2 receptor) and CD28, these findings suggest synergism and cross-talk between the costimulatory pathways.

Different cytokine profiles induced by co-stimulation via anti-CD28 and $I L-2$. The experiment on gene expression profiling was complemented by testing the cytokine content of the supernatants of the different cultures (Table II). Naive T cells activated via CD3 and CD28 produced high amounts of IL-2, IFN- $\gamma$ and TNF- $\alpha$, all Th- 1 cytokines. They also produced the pro-inflammatory cytokine TNF- $\beta$ and the chemokine IP-10. Naive $T$ cells stimulated via CD3/CD25 (using IL-2) also produced increased levels of various cytokines including IFN- $\gamma$ and IP-10 but they did not contain more IL-2 than cultures that were given bsHN-IL2 alone. 


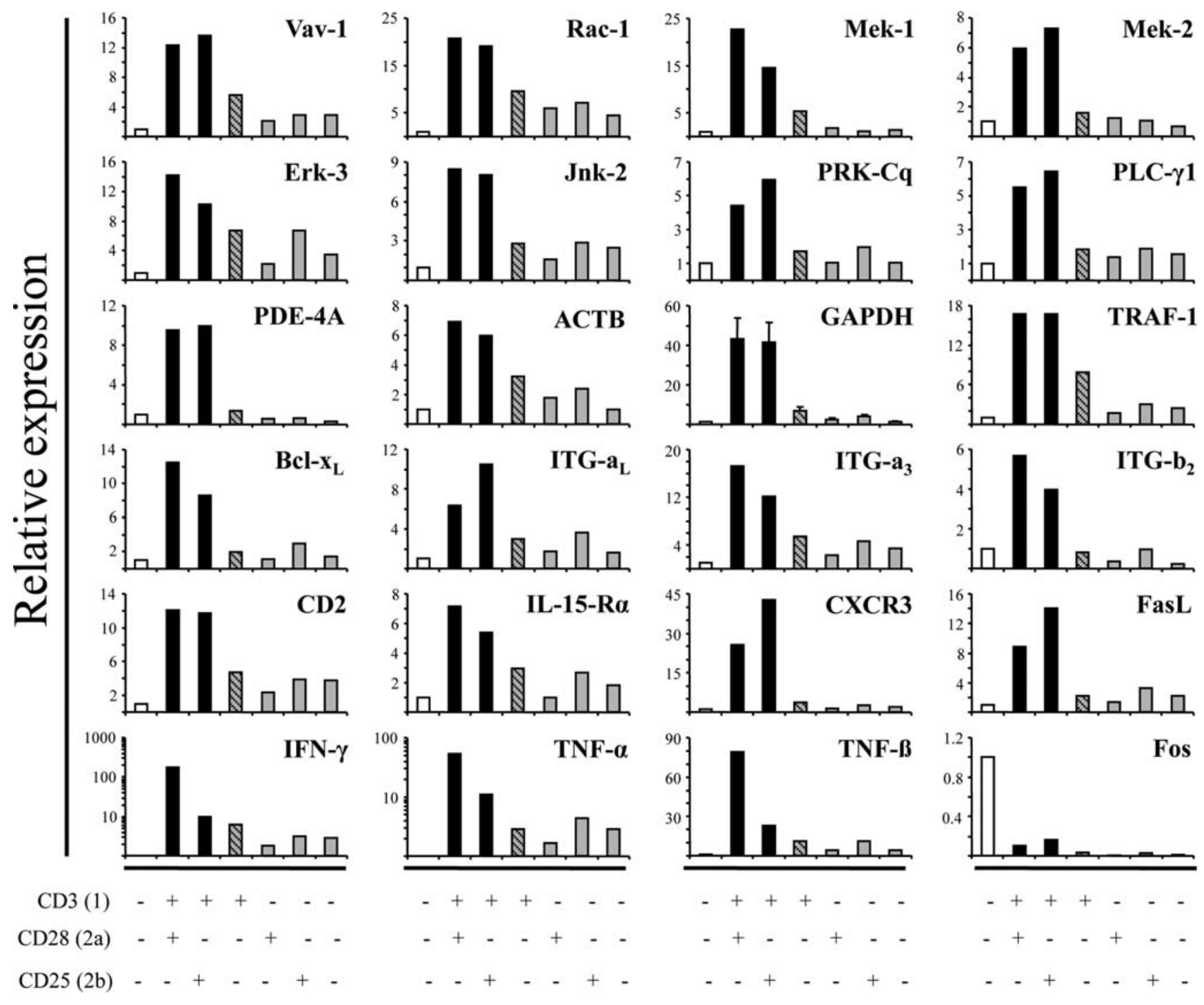

Figure 6. Relative expression of selected genes after CD28 and IL-2 triggering of naive T cells. As in Fig. 5, purified naive T cells were stimulated by $\gamma$-irradiated and NDV-infected MCF-7 tumor vaccine cells (MCF-7-NDV) in the presence of a suboptimal dose of signal 1 (500 pg/well of bsHN-CD3). For the induction of costimulation signals, either bsHN-CD28 or bsHN-IL-2 (black bars, $84 \mathrm{U} /$ well each) were used. Negative controls included naive T cells incubated with MCF-7-NDV cells in the presence of either signal 1 (hatched bars, CD3 alone), costimulatory signal (gray bars, CD28 alone or IL-2 alone), or without any construct (grey bars, vaccine alone). The reference sample comprised unstimulated naive T cells (-) whose gene expression levels was set as $100 \%$ (white bars). After seven days of co-culture, RNA was extracted from the activated T cells, and the array analysis performed as detailed in Materials and methods. A selection of some of the interesting genes is shown (supplemental data for a complete annotated list of genes available from the authors). The data are representative of two independent experiments. Shown are the relative expression levels of fully stimulated naive T cells (CD3/CD28 and CD3/IL-2) and the controls (only CD3: only CD28, only IL-2 and only vaccine) as compared to the 0-value. Gene name: VAV1, Vav-1; Rac-1, RAC1; MAP2K1, Mek-1; MAP2K2, Mek-2; MAPK6, Erk-3; MAPK9, JNK2; PRKCQ, protein kinase C- $\theta$; PLC- $\gamma 1$, phospholipase C- $\gamma$ 1; PDE4A, phosphodiesterase 4A; ACTB, ß-actin; GAPDH, glyceraldehyde-3-phosphate dehydrogenase; TRAF1, TNF receptor-associated factor 1; BCL2L1, bcl-xL; ITG-a $\mathrm{a}_{\mathrm{L}}, \alpha \mathrm{L}$ integrin; ITG-a3, integrin $\alpha 3$; ITG-b2, integrin 32 ; IL15R $\alpha$, IL-15-receptor $\alpha$-chain; CXCR3, chemokine (CXC motif) receptor 3; FASL, Fas ligand; IFN- $\gamma$, interferon gamma; TNF- $\alpha$, TNF alpha, LTA, lymphotoxin $\alpha$ (TNF- $\beta$ ); FOS, Fos.

The differences of cytokine profiles between costimulation via $\mathrm{CD} 28$ and via CD25 were both quantitative and qualitative. The CD25-mediated stimulus induced an 8-10-fold lower level of cytokines and in strong contrast to the CD28-mediated stimulus did not induce an IL-2 response.

The results presented in this study show that vaccine bound IL-2 can induce costimulatory signals via triggering CD25 of naive T cells. There were many similarities to CD28 mediated co-stimulation but also differences. Since we observed synergism and cross-talk between CD25 and CD28 mediated signals, a vaccine providing both costimulatory signals might be optimal.

\section{Discussion}

We demonstrate here that IL-2, expressed as fusion protein (bsHN-IL2) bound to the surface of a virus-modified tumor vaccine is capable of inducing via CD25 a costimulatory signal for $\mathrm{T}$ cell activation.

Preliminary evidence for IL-2-mediated costimulation came from experiments showing induction of proliferation and upregulation of CD25 and CD107a. Vaccine cells modified by bsHN-CD3 (for signal 1) and by bsHN-IL2 induced a similar degree of $\mathrm{T}$ cell activation as the vaccine cells modified with bsHN-CD3 and bsHN-CD28 (for signal 2). Such activated 
Table II. Cytokine secretion profile of activated naïve T cells.

\begin{tabular}{|c|c|c|c|c|c|c|c|c|c|c|c|}
\hline \multirow[b]{2}{*}{ Stimulation } & \multicolumn{11}{|c|}{ Cytokines } \\
\hline & IL-1ß & IL-2 & IL-4 & IL-5 & IL-6 & IL-8 & IL-10 & TNF- $\alpha$ & TNF- $B$ & IFN- $\gamma$ & IP-10 \\
\hline $\begin{array}{l}\mathrm{V}+\mathrm{bsHN}-\mathrm{CD} 3+ \\
\text { bsHN-CD28 }\end{array}$ & 183 & 76,326 & 0 & 0 & 294 & 1,018 & 972 & 670 & 2,135 & 18,379 & 41,720 \\
\hline $\begin{array}{l}\mathrm{V}+\mathrm{bsHN}-\mathrm{CD} 3+ \\
\text { bsHN-IL-2 }\end{array}$ & 9 & 1,537 & 0 & 0 & 149 & 240 & 403 & 96 & 88 & 1,575 & 5,920 \\
\hline $\mathrm{V}+\mathrm{bsHN}-\mathrm{CD} 3$ & 5 & 0 & 0 & 0 & 37 & 0 & 0 & 14 & 16 & 2 & 819 \\
\hline $\mathrm{V}+\mathrm{bsHN}-\mathrm{CD} 28$ & 0 & 0 & 0 & 0 & 19 & 0 & 0 & 0 & 0 & 0 & 204 \\
\hline $\mathrm{V}+\mathrm{bsHN}-\mathrm{IL}-2$ & 0 & 2,344 & 0 & 0 & 21 & 0 & 0 & 0 & 0 & 0 & 382 \\
\hline $\mathrm{V}$ & 0 & 0 & 0 & 0 & 19 & 0 & 0 & 0 & 0 & 0 & 188 \\
\hline
\end{tabular}

Purified naive T cells were activated for seven days as described in Fig. 4. After seven days of activation, the supernatants were removed, centrifuged for $5 \mathrm{~min}$ at 2,600 g and were then analyzed by flow cytometry with the FlowCytomix Multiplex Human Th1/Th2 10plex kit. Only IP-10 was determined by ELISA. All concentrations are given in $\mathrm{pg} / \mathrm{ml}$. The data are representative of two independent experiments.

naive $\mathrm{T}$ cells also showed bystander antitumor effects in an in vitro tumor neutralization assay (TNA).

Kufer et al (26) described a similar approach but they did not use IL-2. They obtained comparable results concerning the activation of naive $\left(\mathrm{CD}_{4} 4 \mathrm{RA}^{+} \mathrm{RO}^{-}\right) \mathrm{T}$ cells by irradiated stimulator cells which were loaded with bispecific singlechain antibodies with anti-CD3 and CD28 ligand specificities. Kufer et al, in contrast to us, did not use a CD28-specific scFv but a B7-containing fusion protein as costimulus. Unlike the $\mathrm{B} 7$ protein, the $\mathrm{scFv} \mathrm{CD} 28$ only binds to $\mathrm{CD} 28$, and not to CTLA-4. Since CTLA-4, which is a late activation antigen of $\mathrm{T}$ cells, mediates $\mathrm{T}$ cell inhibitory signals, stronger and longer-lasting $\mathrm{T}$-cell activation can be expected from the use of our antiCD28 scFv containing fusion proteins.

To define the transcriptome of naive $\mathrm{T}$ cells which were stimulated, we performed a gene chip analysis. As the gene chip contained a total of 247 selected genes, this analysis allowed a more detailed analysis about the co-stimulatory pathway of CD25-mediated costimulatory signaling.

The analyzed genes can be divided into two groups: i) genes for signaling proteins and ii) genes for effector proteins. Signaling occurs a few minutes to several hours after the induction. These signaling proteins are regulated mainly by posttranslational modification such as reversible phosphorylation and through association with secondary 'messenger' molecules $(27,28)$, so that the incoming signal can be rapidly transmitted. The observation that these so-called early events were still measurable after seven days of culture with stimulator cells, led us to conclude that the cells are under constant stimulation which thereby increases the expression of some key signaling proteins. Among them were phospholipase C- $\gamma 1$ (PLC- $\gamma 1)$ and protein kinase $\mathrm{C}-\theta$ $(\mathrm{PKC}-\theta)$, which are the starting point of the NF-AT and $\mathrm{NF}-\kappa \mathrm{B}$ signaling pathway. These were increased only in anti$\mathrm{CD} 3 / \mathrm{CD} 28$ or anti-CD3/IL-2-stimulated naive T cells but not under the control conditions. In addition, the IL-2 receptormediated Jak-STAT signaling cascade was also active (either initiated either by bsHN-IL2 or by endogenous and secreted IL-2).

Proximal events that immediately follow TCR engagement include activation of protein kinases and phosphorylation of multiple enzymes involving scaffold and adaptor molecules (29). The phosphorylation of LAT, a lipid raft-associated adaptor protein, creates docking sites for SH2 domaincontaining proteins thus creating a platform for the recruitment of multiple signalling molecules (30). Three such multiple signalling molecules were found to be highly upregulated according to gene expression profiling upon T cell costimulation with IL-2 immunocytokine-modified vaccine: PLC- $\gamma 1$, the adaptor protein Grb-2 and the Rho-family GTPase exchange factor Vav-1. Anti-CD3/CD25 stimulation also strongly upregulated the expression of the phosphodiesterase type 4A (PDE-4A). These and several other signalling molecules were similarly upregulated in anti-CD3/anti-CD28 and anti-CD3/CD25 stimulated T cells. PLC- $\gamma 1$ recruitment and activation via the LAT-nucleated signalling complex (31) is connected with three signalling pathways which apparently became activated via IL-2-mediated costimulation: i) the MAP kinase (MAPK) pathway, ii) the NF-AT pathway and iii) the NF-кB pathway. In addition to these classical pathways which are also activated via CD28, the IL-2 signalling involves the IL-2 receptor-mediated Jak/STAT pathway $(32,33)$. This leads to the phosphorylation of STAT proteins which also translocate to the nucleus. Our results suggest that costimulation via CD25 on CD3-stimulated TCR signalling networks involves enhanced phosphorylation of PLC- $\gamma 1 / \mathrm{SH} 2$ domain-containing proteins/Grb-2/Vav-1 (34) and their effects on downstream components including MAPKs. Ligation of IL-2 to the heterotrimeric IL-2 receptor activates the Erk type MAPK signalling pathway involving SOS, Ras, Raf, Mek 
costimulus 2b

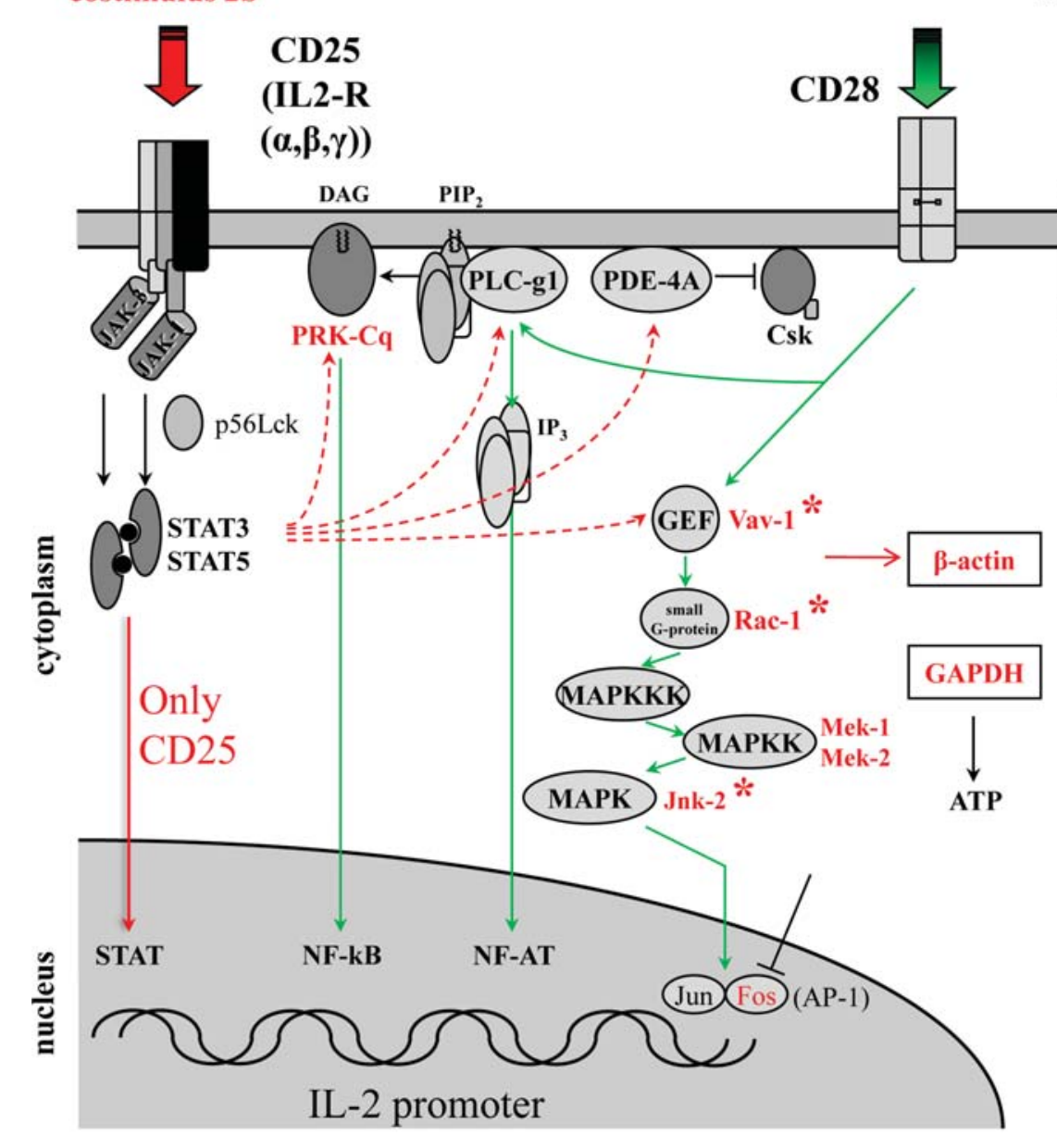

costimulus 2a stimulus 1

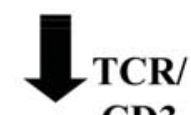

Similar effects of $2 a$ and $2 b$

apoptosis

Bcl- $\mathrm{x}_{\mathrm{L}}$; Caspase-9

cell adhesion

Integrin $\mathrm{a}_{\mathrm{L}_{i}}$ Integrin $\mathrm{a}_{3}$ Integrin $\mathrm{b}_{2} \mathrm{CD} 2$

receptors / ligands

TNFSF7 (CD70);

TNF- $\alpha / \beta$ receptor

TNFRSF7 (CD27);

Fas-Ligand (CD95L)

IL-10 receptor (a-chain);

IL-12 receptor (b2-chain)

IL-15 receptor (a-chain);

CD81 CTLA-4; CCR5;

CXCR3;

CD40-Ligand ; (CD154)

cytokines / chemokines

IP-10; IL-13; IFN- $\gamma$;

GM-CSF; TNF- $\alpha$; TNF-B

Different effects

of $2 a$ and $2 b$

Figure 7. Model of activation of naive T cells by the three following signals: CD3, CD28 and IL-2 showing synergism and cross-talk between the two costimuli anti-CD28 (2a) and IL-2 (2b). In the presence of signal 1 (TCR:CD3 engagement), anti-CD28 (signal 2a) and IL-2 (signal 2b) induce largely overlapping patterns of gene expression. Intracellular proteins (mostly signalling molecules) that are equally upregulated by both costimuli are shown in red (rigth side). Signal 1 and costimulatory signals alter the cytoskeleton to produce changes in cell shape, motility and secretion. Upregulated effector genes are shown on the right side (classified by function). Both costimuli induce the NF-kB and NF-AT pathway via the key enzymes PKC- $\theta$ and PLC- $\gamma 1$, respectively. The IL-2 receptor-mediated Jak/STAT signalling pathway is switched on due to the IL-2 in the supernatant (either in form of exogenously added bsHN-IL-2 or in form of endogenously secreted IL-2: see also Table II). Note that signalling molecules of the CD28-mediated stress-activated protein kinase (Sapk) pathway, namely Vav-1, Rac-1 and Jnk-2 (marked by an asterisk), are also upregulated by triggering via CD25 by bsHN-IL2. ( $\rightarrow$ ) direction of signal flow; (-I) interruption of

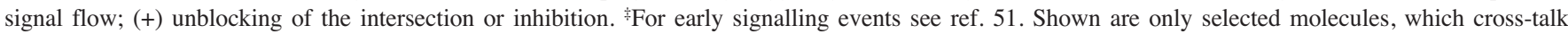
between the IL-2 and the CD28 signaling pathways. Red marked molecules and those indicated at the right side are increased among conditions of full activation with CD3/CD28 or CD3/IL-2 compared to controls. The signal molecules which are marked with an asterisk (*) of the MAP kinase cascade are specific to costimulation. See text for more details. Abbreviations: AP-1, activated protein 1; ATP, adenosine tri-phosphate; CCR5, CC chemokine receptor 5; Csk, C-terminal Src kinase; CTLA-4, cytotoxic T lymphocyte-associated protein 4; DAG, diacylglycerol; GEF, guanine nucleotide exchange factor; GM-CSF, granulocyte/macrophage colony stimulating factor; Grb-2, growth factor receptor-bound protein 2; IL-2-R $(\alpha / \beta / \gamma c)$, heterotrimeric high-affinity IL-2 receptor; IP-10, IFN- $\gamma$ inducible protein of $10 \mathrm{kDa}$; IP3, inositol-1,4,5-triphosphate; Jak, Janus kinase; MAPKK, MAPK kinase; MAPKKK, MAPKK kinase; NF-AT, nuclear factor of activated T cells; NF-кB, nuclear factor kappa B; PIP2, phosphatidylinositol-4,5-bisphosphate; STAT, signal transducer and activator of transcription; TCR/CD3, T cell receptor, CD3 complex (with the ITAM regions shown in yellow); TNFRSF, TNF receptor superfamily; TNFSF, TNF superfamily. For further abbreviations see Fig. 6 and text.

and Erk (17). Our data suggest for the first time that IL-2 can also lead to the activation of the Sapk type MAPK signalling pathway (35) and to the activation of c-Jun.

IL-2 has an important influence on the MAPK signalling pathway. For T-cells, there are two different MAPK signaling cascades described: either the Erk-type (Erk, extracellular regulated kinase) or the SAPK-type (SAPK, stress-activated protein kinase) (35). It has been described that the ligation of IL-2 to the heterotrimeric IL-2 receptor can induce via SOS (a nucleotide exchange factor), Ras (a small G-protein), Raf, Mek and Erk the Erk-type signalling pathway (17). This was found to be true also for bsHN-IL2. In addition, we observed induction of the SAPK-type signaling pathway by IL-2 in our gene chip experiment which has not been described up to now. This pathway is so far only known for CD3/CD28 fully activated $\mathrm{T}$ cells and leads to the stimulation of Jnk $(36,37)$ and also of Jun, which, along with Fos in the Erk signaling pathway, forms the transcription factor AP-1 which is important for activation of T-cells.

Anti-CD3/IL-2 stimulated upregulation of genes for effector proteins including cell adhesion molecules such as LFA-1 and CD2, members of the TNF and TNF receptor 
superfamilies such as CD27, CD70, CD40 ligand, Fas ligand, TNF- $\alpha$ and TNF- $\beta$ as well as the corresponding receptors. Among apoptosis-related genes we saw an upregulation of the anti-apoptotic gene bcl-xL as well as an upregulation of the pro-apoptotic gene caspase 9 . The latter, together with the observed upregulation of CTLA-4, might be interpreted as an indication of cellular events occurring seven days after stimulation which are aiming at termination of the response. We furthermore observed, after anti-CD3/IL-2 stimulation, increased levels of IFN- $\gamma$ and IP-10 and to some extent IL-10 in the supernatants. The gene chip analysis revealed also an upregulation of the receptor for IP-10 (CXCR3), of the IL-12 receptor $\alpha$-chain and of the IL-15 receptor $\alpha$-chain. These latter changes are often seen in close association with $\mathrm{T}$ cell activation in general, with $\mathrm{T}$ cell proliferation and with the generation of memory $\mathrm{T}$ cells (38). It is also of interest to mention the upregulation of $B$-actin (ACTB) which is required for organisation of the cytoskeleton and the 40-fold upregulation of the metabolic enzyme GAPDH which provides ATP for the increased metabolism of the activated cells.

In general, the gene chip analysis showed that freshly isolated naive $\mathrm{T}$ cells in the presence of a suboptimal signal 1 (bsHN-CD3) could become costimulated via CD25. Similar genes were upregulated after seven days as in $\mathrm{T}$ cells that were activated in a classical way via anti-CD3 and anti-CD28. Controls, which had been stimulated with signal 1 or signal 2 alone, showed only marginal responses.

One reason for the similarity in the mode of action of IL-2and CD28-mediated signals could be the tyrosine kinase p56Lck (Lck) which is associated both with the $B$ chain of IL-2 receptor (32) and with the TCR-associated co-receptors CD4 and CD8 (39). This would also explain why other costimulatory molecules such as CD2 do not lead to the activation of the SAPK-type pathway (35).

Since the two costimuli CD28 and IL-2 seem to use similar signaling pathways in the initiation of a T-cell response, it is not surprising that genes coding for effector proteins, were also similarly upregulated. This was the case for adhesion molecules, the TNF and the TNF receptor superfamily and for regulators of apoptosis and of energy metabolism.

The two co-stimulatory signals mediated either via CD28 or via CD25, however, differed in one aspect, the production of IL-2. While naive T cells activated via CD3/CD28 are capable of secreting IL-2 in large quantities, this was not seen with naive $\mathrm{T}$ cells activated via $\mathrm{CD} 3 / \mathrm{CD} 25$. Through stimulation with bsHN-IL2, the naive T cells were not capable of producing IL-2. Similar observations were made by Yashiro et al (40) who used mAbs against CD5, CD9, CD2, CD44 and CD11a in the presence of a suboptimal anti-CD3 signal. These stimuli induced a proliferation of naive $\mathrm{T}$ cells after $48 \mathrm{~h}$ comparable to the one induced via CD28. However, in comparison to the T-cell response mediated by CD28 co-stimulation, the $\mathrm{T}$ cell responses mediated by other costimulation molecules led to a decrease in viable cell count and to apoptosis induction. These effects were associated with an inability of these $\mathrm{T}$ cells to produce IL-2. An additional administration of recombinant IL-2 to the culture medium prevented the induction of apoptosis (40). Since exogenous IL-2 is present during T-cell stimulation through the construct bsHN-IL2, CD3/CD25-activated naive T cells should be resistant to apoptosis. The anti-apoptotic protein bcl-xL was highly upregulated in such stimulated cells (41). In addition, propidium iodide-staining of CD3/CD25-stimulated cells never showed in FACS a higher percentage of dead cells as compared to $\mathrm{CD} 3 / \mathrm{CD} 28$-stimulated cells (data not shown). Thus, IL-2 apparently has a dual role via CD25: first it acts in conjunction with the CD3 signal as a costimulus (such as CD28) and secondly, in later stages of activation, as a classic, but integral T-cell growth factor, it supports the expansion of the cells and suppresses apoptosis (42). In this respect, the IL-2 signal seems to be superior to all other non-CD28costimuli so far described.

One explanation for the differential capacity for IL-2 production of differently activated naive $\mathrm{T}$ cells could be the complex structure of the promoter region of the IL-2 gene. This has binding sites for the previously mentioned transcription factors NF-AT, NF- $\mathrm{kB}$ and AP-1, and contains CD28RE (CD28 responsive element), a DNA sequence, to which the CD28RC (CD28 responsive complex) binds $(43,44)$. $\mathrm{CD} 28 \mathrm{RE}$ is a regulatory sequence for the full induction of the IL-2 promoter. It plays a crucial role for specific transcription factors - e.g., specific members of the NF- $\mathrm{BB}$ family such as c-Rel. These are recruited after a B7-1/CD28-stimulus, but not after a non-CD28 stimulus $(35,45)$. It is possible that a main effect of CD28 costimulation consists in the loosening of the nucleosomal DNA of the promoter/enhancer region of the IL-2 gene by targeted histone acetylation and demethylation of $\mathrm{CpG}$ dinucleotides (46). It would thus be conceivable that, in our analysis, the signaling pathway induced by IL-2 does not lead to the opening of the compact chromatin structure and/or to the binding of essential transcription factor complexes in the promoter/enhancer-region of the IL-2 promoter.

However, it was demonstrated that $\mathrm{HN}$ molecules when expressed at the surface of NDV-infected tumor vaccine induce a B7-1/B7-2-independent costimulation effect leading to induction of CD28RC in T lymphocytes $(23,47)$. These observations differ from the present results obtained with naive T-cells in so far as Termeer et al (47) and Ertel et al (23) used, in their studies, memory T cells. These cells can be activated, in contrast to naive $\mathrm{T}$ cells, even with a much weaker costimulus. It is then possible that the strength of the co-stimulatory signal (mediated by the HN molecules on the surface of the vaccine cells) was sufficient to activate memory T-cells. But the HN-costimulatory signal is probably not sufficient in strength to lead to the activation of naive $\mathrm{T}$ cells. Overall, these data demonstrate that the response of naive $\mathrm{T}$ cells leading to IL-2 production is highly complex. Further experiments are needed to identify the exact mechanisms of signal transduction pathways in CD3/CD25-activated naive $\mathrm{T}$ cells and to define the nature of transcription factors at the IL-2-locus involved in activation and proliferation of naive and memory $\mathrm{T}$ cells.

In Fig. 7 we present the possible signalling pathways and effects induced by the various stimuli. We are focussing on late events since the analysis was performed at day seven after activation. Nine of the genes that were strongly upregulated are shown in red. They belong to the MAP kinase pathway, to the phospholipase C (PLC)- $\gamma 1$ pathway or to the protein kinase C- $\theta$ pathway. Just as anti-CD28, IL-2 via CD25 apparently can function as a costimulus by being 
able to activate all these three signalling pathways. In addition there is the IL-2 receptor-dependent Jak/STAT pathway. Of particular significance for $\mathrm{T}$ cell costimulation are the molecules Vav-1 and Rac-1. They are linked to B-actin, cytoskeleton reorganisation, cell migration and, in tumor cells, to invasion and metastasis $(48,49)$. The figure also summarizes the effects of the upregulated genes on the cells' function. The effects involve apoptosis, cell adhesion, receptors/ligands and cytokines/chemokines. The main difference between costimulation via CD25 and CD28, as highlighted by the arrow in red, concerns the IL-2 response.

Noteworthy is that the activation of naive $\mathrm{T}$ cells by our vaccines loaded with bsAbs or immuno-cytokines is Th1 polarized leading to effector funcions such as cytoxicity and IFN- $\gamma$ production. This can explain the strong bystander antitumor effects observed in the TNA.

In addition, fully activated naive $\mathrm{T}$ cells after five to seven days of exposure to the modified tumor vaccines, express the memory $\mathrm{T}$ cell marker CD45RO (26). Thus, our fusion proteins in combination with the tumor vaccine ATV$\mathrm{NDV}$, are in a position to induce an immunological $\mathrm{T}$ cell memory. That is of clinical and therapeutic relevance.

In conclusion, we discovered in this study that during priming of naive $\mathrm{T}$ cells with a second generation tumor vaccine, the new IL-2 immunocytokines provided classical $\mathrm{T}$ cell costimulatory activity. Stimulation of naive $\mathrm{T}$ cells with tumor vaccine exposing anti-CD3 and IL-2 initiated a cascade of molecular events leading to $\mathrm{T}$ cell activation, proliferation and cytokine production. Our data demonstrate that IL-2 participates in the activation of naive T cells as costimulus in a similar but also distinct way as compared to CD28. This could be used for potentiation and broadening of the application of the ATV-NDV tumor vaccine which already has shown in various tumor entities (50) promising results in clinical studies.

\section{Acknowledgements}

This project is part of the $\mathrm{PhD}$ thesis of Maximilian Aigner under co-supervision by Professor Volker Schirrmacher and Dr Philippe Fournier. We are particularly grateful to Annette Arnold for technical assistance. The writing of this manuscript would not have been possible without the kind financial support of the Dr Kleist Stiftung (Berlin, Germany) and the Christian-Berndt Stiftung (Rostock, Germany). We gratefully acknowledge also support by the IOZK (www.iozk.de) (Cologne, Germany).

\section{References}

1. Feuerer M, Beckhove P, Bai L, Solomayer EF, Bastert G, Diel IJ, Pedain C, Oberniedermayr M, Schirrmacher V and Umansky V: Therapy of human tumors in NOD/SCID mice with patient-derived reactivated memory $\mathrm{T}$ cells from bone marrow. Nat Med 7: 452-458, 2001.

2. Galon J, Costes A, Sanchez-Cabo F, Kirilovsky A, Mlecnik B, Lagorce-Pagès $\mathrm{C}$, Tosolini $\mathrm{M}$, Camus $\mathrm{M}$, Berger $\mathrm{A}$, Wind $\mathrm{P}$, Zinzindohoué F, Bruneval P, Cugnenc PH, Trajanoski Z, Fridman WH and Pagès F: Type, density, and location of immune cells within human colorectal tumors predict clinical outcome. Science 313: 1960-1964, 2006

3. Seliger B, Maeurer MJ and Ferrone S: Antigen-processing machinery breakdown and tumor growth. Immunol Today 21 $455-464,2000$
4. Hicklin DJ, Marincola FM and Ferrone S: HLA class I antigen downregulation in human cancers: T-cell immunotherapy revives an old story. Mol Med Today 5: 178-186, 1999.

5. Sharpe AH and Freeman GJ: The B7-CD28 superfamily. Nat Rev Immunol 2: 116-126, 2002.

6. Kim R, Emi M, Tanabe K and Arihiro K: Tumor-driven evolution of immunosuppressive networks during malignant progression. Cancer Res 66: 5527-5536, 2006.

7. Hellstrom KE and Hellstrom I: Novel approaches to therapeutic cancer vaccines. Expert Rev Vaccines 2: 517-532, 2003.

8. Turk MJ, Guevara-Patiño JA, Rizzuto GA, Engelhorn ME, Sakaguchi S and Houghton AN: Concomitant tumor immunity to a poorly immunogenic melanoma is prevented by regulatory T cells. J Exp Med 200: 771-782, 2004

9. Chen L, Linsley PS and Hellstrom KE: Costimulation of T cells for tumor immunity. Immunol Today 14: 483-486, 1993.

10. June CH, Ledbetter JA, Linsley PS and Thompson CB: Role of the CD28 receptor in T-cell activation. Immunol Today 11: 211-216, 1990 .

11. Fraser JD, Irving BA, Crabtree GR and Weiss A: Regulation of interleukin-2 gene enhancer activity by the $\mathrm{T}$ cell accessory molecule CD28. Science 251: 313-316, 1991.

12. Jenkins MK, Taylor PS, Norton SD and Urdahl KB: CD28 delivers a costimulatory signal involved in antigen-specific IL-2 production by human T cells. J Immunol 147: 2461-2466, 1991.

13. Lenschow DJ, Walunas TL and Bluestone JA: CD28/B7 system of T cell costimulation. Annu Rev Immunol 14: 233$258,1996$.

14. Zambricki E, Shigeoka A, Kishimoto H, Sprent J, Burakoff S, Carpenter C, Milford E and McKay D: Signaling T-cell survival and death by IL-2 and IL-15. Am J Transplant 5: 2623-2631, 2005.

15. Tibaldi EV, Salgia R and Reinherz EL: CD2 molecules redistribute to the uropod during $\mathrm{T}$ cell scanning: implications for cellular activation and immune surveillance. Proc Natl Acad Sci USA 99: 7582-7587, 2002

16. Yusuf-Makagiansar H, Anderson ME, Yakovleva TV, Murray JS and Siahaan TJ: Inhibition of LFA-1/ICAM-1 and VLA-4/ VCAM-1 as a therapeutic approach to inflammation and autoimmune diseases. Med Res Rev 22: 146-167, 2002.

17. Gaffen SL: Signaling domains of the interleukin 2 receptor. Cytokine 14: 63-77, 2001

18. Maes BD and Vanrenterghem YF: Anti-interleukin-2 receptor monoclonal antibodies in renal transplantation. Nephrol Dial Transplant 14: 2824-2826, 1999.

19. Demaison C, Chastagner P, Moreau JL and Theze J: Ligandinduced autoregulation of IL-2 receptor alpha chain expression in murine T cell lines. Int Immunol 8: 1521-1528, 1996.

20. Fiola C, Peeters B, Fournier P, Arnold A, Bucur M and Schirrmacher V: Tumor selective replication of Newcastle disease virus: association with defects of tumor cells in antiviral defence. Int J Cancer 119: 328-338, 2006.

21. Schirrmacher V, Haas C, Bonifer R, Ahlert T, Gerhards R and Ertel C: Human tumor cell modification by virus infection: an efficient and safe way to produce cancer vaccine with pleiotropic immune stimulatory properties when using Newcastle disease virus. Gene Ther 6: 63-73, 1999.

22. Washburn B and Schirrmacher V: Human tumor cell infection by Newcastle Disease Virus leads to upregulation of HLA and cell adhesion molecules and to induction of interferons, chemokines and finally apoptosis. Int J Oncol 21: 85-93, 2002.

23. Ertel C, Millar NS, Emmerson PT, Schirrmacher V and von Hoegen P: Viral hemagglutinin augments peptide-specific cytotoxic T cell responses. Eur J Immunol 23: 2592-2596, 1993.

24. Haas C, Lulei M, Fournier P, Arnold A and Schirrmacher V: T-cell triggering by CD3- and CD28-binding molecules linked to a human virus-modified tumor cell vaccine. Vaccine 23 : 2439-2453, 2005.

25. Bian H, Fournier P, Moormann R, Peeters B and Schirrmacher V: Selective gene transfer in vitro to tumor cells via recombinant Newcastle disease virus. Cancer Gene Ther 12: 295-303, 2005.

26. Kufer P, Zettl F, Borschert K, Lutterbuse R, Kischel R and Riethmuller G: Minimal costimulatory requirements for $\mathrm{T}$ cell priming and TH1 differentiation: activation of naive human T lymphocytes by tumor cells armed with bifunctional antibody constructs. Cancer Immun 1: 10, 2001. 
27. Mann M, Ong SE, Gronborg M, Steen H, Jensen ON and Pandey A: Analysis of protein phosphorylation using mass spectrometry: deciphering the phosphoproteome. Trends Biotechnol 20: 261-268, 2002.

28. Steen H, Pandey A, Andersen JS and Mann M: Analysis of tyrosine phosphorylation sites in signaling molecules by a phosphotyrosine-specific immonium ion scanning method. Sci STKE. PL16, 2002.

29. Geng L and Rudd CE: Signalling scaffolds and adaptors in T-cell immunity. Br J Haematol 116: 19-27, 2002.

30. Wange RL: LAT, the linker for activation of T cells: a bridge between $\mathrm{T}$ cell-specific and general signaling pathways. Sci STKE 2000: 63, 2000

31. Braiman A, Barda-Saad M, Sommers CL and Samelson LE: Recruitment and activation of PLCgamma1 in T cells: a new insight into old domains. EMBO J 25: 774-784, 2006.

32. Hatakeyama M, Kono T, Kobayashi N, Kawahara A, Levin SD, Perlmutter RM and Taniguchi T: Interaction of the IL-2 receptor with the src-family kinase p56lck: identification of novel intermolecular association. Science 252: 1523-1528, 1991.

33. Tigges MA, Casey LS and Koshland ME: Mechanism of interleukin-2 signaling: mediation of different outcomes by a single receptor and transduction pathway. Science 243: 781-786, 1989

34. Ardouin L, Bracke M, Mathiot A, Pagakis SN, Norton T, Hogg $\mathrm{N}$ and Tybulewicz VL: Vav1 transduces TCR signals required for LFA-1 function and cell polarization at the immunological synapse. Eur J Immunol 33: 790-797, 2003.

35. Visse E, Inostroza J, Cabello G and Parra E: The MAP kinases are differently utilized by CD28 and CD2 adhesion pathways in superantigen-activated Jurkat T cells. Biol Res 36: 263-278, 2003.

36. Su B, Cheng J, Yang J and Guo Z: MEKK2 is required for T-cell receptor signals in JNK activation and interleukin-2 gene expression. J Biol Chem 276: 14784-14790, 2001.

37. Minden A, Lin A, Claret FX, Abo A and Karin M: Selective activation of the JNK signaling cascade and c-Jun transcriptional activity by the small GTPases Rac and Cdc42Hs. Cell 81: 1147-1157, 1995.

38. Sallusto F, Geginat $\mathrm{J}$ and Lanzavecchia A: Central memory and effector memory $\mathrm{T}$ cell subsets: function, generation, and maintenance. Annu Rev Immunol 22: 745-763, 2004.

39. Reedquist KA, Fukazawa T, Druker B, Panchamoorthy G, Shoelson SE and Band H: Rapid T-cell receptor-mediated tyrosine phosphorylation of p120, an Fyn/Lck Src homology 3 domain-binding protein. Proc Natl Acad Sci USA 91: 4135-4139, 1994.

40. Yashiro Y, Tai XG, Toyo-oka K, Park CS, Abe R, Hamaoka T, Kobayashi M, Neben S and Fujiwara H: A fundamental difference in the capacity to induce proliferation of naive $\mathrm{T}$ cells between CD28 and other co-stimulatory molecules. Eur J Immunol 28: 926-935, 1998.
41. Akbar AN, Borthwick NJ, Wickremasinghe RG, Panayoitidis P, Pilling D, Bofill M, Krajewski S, Reed JC and Salmon M: Interleukin-2 receptor common gamma-chain signaling cytokines regulate activated $\mathrm{T}$ cell apoptosis in response to growth factor withdrawal: selective induction of anti-apoptotic (bcl-2, bclxL) but not pro-apoptotic (bax, bcl-xS) gene expression. Eur J Immunol 26: 294-299, 1996.

42. D'Souza WN and Lefrançois L: IL-2 is not required for the initiation of CD8 $\mathrm{T}$ cell cycling but sustains expansion. J Immunol 171: 5727-5735, 2003.

43. Parra E, Varga M, Hedlund G, Kalland T and Dohlsten M: Costimulation by B7-1 and LFA-3 targets distinct nuclear factors that bind to the interleukin-2 promoter: B7-1 negatively regulates LFA-3-induced NF-AT DNA binding. Mol Cell Biol 17: 1314-1323, 1997

44. Jain J, Valge-Archer VE, Sinskey AJ and Rao A: The AP-1 site at $-150 \mathrm{bp}$, but not the NF-kappa B site, is likely to represent the major target of protein kinase $\mathrm{C}$ in the interleukin 2 promoter. $\mathrm{J}$ Exp Med 175: 853-862, 1992.

45. Zhou XY, Yashiro-Ohtani Y, Nakahira M, Park WR, Abe R, Hamaoka T, Naramura M, Gu H and Fujiwara H: Molecular mechanisms underlying differential contribution of CD28 versus non-CD28 costimulatory molecules to IL-2 promoter activation. J Immunol 168: 3847-3854, 2002.

46. Thomas RM, Gao L and Wells AD: Signals from CD28 induce stable epigenetic modification of the IL-2 promoter. J Immunol 174: 4639-4646, 2005.

47. Termeer CC, Schirrmacher V, Brocker EB and Becker JC: Newcastle disease virus infection induces B7-1/B7-2-independent T-cell costimulatory activity in human melanoma cells. Cancer Gene Ther 7: 316-323, 2000.

48. Puhlmann J, Puehler F, Mumberg D, Boukamp P and Beier R: Rac1 is required for oncolytic NDV replication in human cancer cells and establishes a link between tumorigenesis and sensitivity to oncolytic virus. Oncogene 29: 2205-2216, 2010.

49. Kimmelman AC, Hezel AF, Aguirre AJ, Zheng H, Paik JH, Ying H, Chu GC, Zhang JX, Sahin E, Yeo G, Ponugoti A, Nabioullin R, Deroo S, Yang S, Wang X, McGrath JP, Protopopova M, Ivanova E, Zhang J, Feng B, Tsao MS, Redston M, Protopopov A, Xiao Y, Futreal PA, Hahn WC, Klimstra DS, Chin L and De Pinho RA: Genomic alterations link Rho family of GTPases to the highly invasive phenotype of pancreas cancer. Proc Natl Acad Sci USA 105: 19372-19377, 2008.

50. Schirrmacher V and Fournier P: Newcastle disease virus: a promising vector for viral therapy, immune therapy, and gene therapy of cancer. Methods Mol Biol 542: 565-605, 2009.

51. Samelson LE: Signal transduction mediated by the $T$ cell antigen receptor: the role of adapter proteins. Annu Rev Immunol 20: 371-394, 2002. 